\title{
Energy Dependent Transport Model of the Neutron Number Probability Distribution in a Subcritical Multiplying Assembly
}

\author{
J. E. M. Saxby ${ }^{1}$, Anil K. Prinja ${ }^{1,2}$, M. D. Eaton ${ }^{1}$ \\ ${ }^{1}$ Nuclear Engineering Group, Department of Mechanical Engineering, Imperial College London, \\ Exhibition Road, London SW7 2AZ, UK \\ ${ }^{2}$ Department of Nuclear Engineering, University of New Mexico, Albuquerque, NM 87131, USA
}

(c) British Crown Owned Copyright 2017/AWE

\begin{abstract}
The time and phase-space dependent backward Master equation is used to develop and numerically solve a coupled system of transport equations for the probability distribution of the neutron number in subregions of a spherically symmetric, reflected, subcritical plutonium sphere. The number distributions are computed for a single initial neutron injected into the assembly and localised in phase space as well as in the presence of a uniformly distributed spontaneous fission source in the fissile region. A standard multigroup, discrete ordinates scheme with second order spatial and fully implicit time discretisation proved sufficiently accurate for this application. The results presented show complex behaviours arising from the material interface and spectral effects due to neutron slowing down that cannot be encapsulated in a lumped model. Additionally, low order spatial moments were computed both by averaging the number distributions of finite order and directly solving the transport equations for the moments using the same numerical scheme. While generally excellent agreement is observed between the two approaches, the truncation order has a noticeable effect on the accuracy of the higher moments that are computed using the number distributions.
\end{abstract}

\section{Introduction}

The ability to model and measure neutron multiplicity distributions plays an important role in characterising small samples of special nuclear material that is of particular relevance in nuclear nonproliferation and safeguards research. Neutrons released in spontaneous fission reactions in fissile materials can induce further fissions and the resulting multiplicity distribution provides a unique and characteristic signature of the multiplying medium that is distinct from that of background neutrons, such as that arising from $(\alpha, \mathrm{n})$ reactions. The measured or computed multiplicities can then be used to infer gross properties of the material in question, specifically its mass and isotropic composition $[1,2]$.

Email address: j.saxby12@imperial.ac.uk (J. E. M. Saxby $\left.{ }^{1}\right)$ 
To date, computational methods have focussed on low order statistical moments, for which deterministic transport codes can be readily adapted, or have alternatively used Monte Carlo codes to predict the entire neutron probability distribution by simulating large numbers of neutron histories. The first method provides gross statistical properties such as the mean, variance, skewness and kurtosis of a neutron number distribution, for comparison to the measured statistics [3] and the second is highly computationally expensive, although it is valuable for benchmarking results obtained using simpler approaches. However, when the multiplication due to induced fission in a sample is weak such that the neutron population is not large, a third approach becomes feasible: the direct numerical solution of the backward Master equation to obtain the probability distribution of neutron numbers. This formulation yields a closed, coupled set of differential equations for the number probabilities corresponding to successively higher numbers of neutrons and must be solved sequentially. For small, highly subcritical samples - where neutron populations extend only into the tens - this approach has the potential to be very efficient while enabling essential details of physics and geometry to be captured. Despite this, relatively little computational work has been done on calculating the neutron number probabilities in this manner. Until recently, numerical work based on Master equations had been restricted to the solution of point or lumped models for a single source realisation [2] for both neutrons and photons. Whilst point models are useful for developing physical understanding and intuition, they cannot quantify any spatial effects which, in the case of multilayered media, are especially vital. A generalisation of this approach to include spatial dependence using a one-speed diffusion approximation of the backward transport equation has been recently described by the authors [4], which clearly shows the importance of including spatial dependence in computational investigations. Whilst this was a valuable and interesting extension to work previously undertaken, and not an unreasonable approach for large, fast systems, a more complete and accurate approach to computing the neutron number distributions must be based on the space-angle-energy, or phase-space, dependent backward Master equation. This is especially true for the small samples typically considered in nuclear security scenarios where boundaries strongly influence the angular distribution and the presence of moderating material causes a noticeable spectral shift. We note that the effect of energy dependence on the survival probability (the complement of the extinction probability) has been considered previously [5] but without accounting for temporal or spatial dependence and moreover focussed only on super-critical systems. Also, methods based on numerical inversion of the generating function have been reported $[6,7,8]$ to calculate number distributions, but thus far, these have been restricted to the point model and the efficacy of the approach when the neutron space, energy and angle dependence is included has yet to be established.

In this paper, we extend our previous work [4] and demonstrate that it is feasible to compute the neutron number probabilities while accounting for temporal, spatial, energy and angular variation of the temporally evolving and random neutron population under the circumstances described above. We base our approach on the general nonlinear transport equation for the generating function, as articulated in [9], and numerically solve for the number probabilities for both a single initiating neutron and a randomly 
emitting source within a fissile material. We specifically apply our methodology to a one-dimensional, spherically symmetric assembly comprising a reflected plutonium sphere and use standard transport discretisation methods for the numerical solution: discrete ordinates in angle, multigroup in energy, second order finite difference in space, and fully implicit in time. We also derive and solve, using the same numerical scheme, a coupled set of linear transport equations for a number of low order factorial moments, which directly yield the mean, variance, etc., of the neutron distribution and provide a complementary characterisation of the neutron population. Additionally, these directly computed statistical moments are compared to the moments obtained by ensemble averaging over the numerically computed number probabilities to provide an independent verification of the accuracy of the solution of the number probabilities. Extensive numerical results for neutron number probabilities of various orders and the statistical moments in the reflected geometry, without and with an intrinsic random source, are presented. Our results show unique features that can be attributed to the presence of boundary layers at spatial interfaces and at the outer surface, and to spectral shifts arising from neutron slowing down.

The scope of the paper is as follows: the general formulation in terms of the generating function, for a single initiating neutron and a random intrinsic source, is developed in the next section followed by a derivation of the coupled equations for the number probabilities. The model is then specialised to a one dimensional, multilayered spherical system, the numerical solution is briefly presented and the numerical results are discussed in some detail. The paper concludes with some final remarks and an appendix providing details of the derivation of the number probability equations.

\section{Backward Master Equation Formulation}

We consider the evolution of the neutron population in a convex multiplying body of volume $V \subset \mathbb{R}^{3}$ and bounding surface $\partial V \subset \mathbb{R}^{2}$ subject to scattering, capture and fission events. The neutron phase space is characterised by the spatial position $\vec{r} \in V$, energy $E \in \mathbb{E} \subset \mathbb{R}$ and the direction of travel $\vec{\Omega} \in S^{2}$ on the unit sphere. We define $p_{n}\left(R, t_{f} ; \vec{r}, E, \vec{\Omega}, t\right), n=0,1,2 \ldots$, as the probability of finding $n$ neutrons in the phase space sub-volume $R \subset V \times S^{2} \times \mathbb{E}$ at a time $t_{f}$, given that one neutron was injected into the medium at an earlier time, $t$, at position $\vec{r}$, with energy $E$ and traveling in direction $\vec{\Omega}$. The random process describing the neutron population is Markovian and under these conditions $p_{n}\left(R, t_{f} ; \vec{r}, E, \vec{\Omega}, t\right)$ is known to satisfy the backward Master equation $[10,9,11]$. In terms of the generating function defined by [9]:

$$
G\left(R, z, t_{f} ; \vec{r}, E, \vec{\Omega}, t\right)=\sum_{n=0}^{\infty} z^{n} p_{n}\left(R, t_{f} ; \vec{r}, E, \vec{\Omega}, t\right),
$$

or, for our purposes, the modified generating function:

$$
\tilde{G}\left(R, z, t_{f} ; \vec{r}, E, \vec{\Omega}, t\right)=1-G\left(R, z, t_{f} ; \vec{r}, E, \vec{\Omega}, t\right)
$$


the backward Master equation can be transformed into the more compact and convenient form given by [9]:

$$
\begin{aligned}
& -\frac{1}{v} \frac{\partial \tilde{G}\left(R, z, t_{f} ; \vec{r}, E, \vec{\Omega}, t\right)}{\partial t}-\vec{\Omega} \cdot \nabla \tilde{G}\left(R, z, t_{f} ; \vec{r}, E, \vec{\Omega}, t\right)+\Sigma_{t}(\vec{r}, E, t) \tilde{G}\left(R, z, t_{f} ; \vec{r}, E, \vec{\Omega}, t\right) \\
& \quad=\iint \Sigma_{s}\left(\vec{r}, E \rightarrow E^{\prime}, \vec{\Omega} \cdot \overrightarrow{\Omega^{\prime}}, t\right) \tilde{G}\left(R, z, t_{f} ; \vec{r}, E^{\prime}, \vec{\Omega}^{\prime}, t\right) d \vec{\Omega}^{\prime} d E^{\prime} \\
& \quad+\bar{\nu}(\vec{r}, E, t) \Sigma_{f}(\vec{r}, E, t)\left[\iint F\left(E^{\prime}\right) \tilde{G}\left(R, z, t_{f} ; \vec{r}, E^{\prime}, \vec{\Omega}^{\prime}, t\right) \frac{d \vec{\Omega}^{\prime}}{4 \pi} d E^{\prime}\right] \\
& \quad-\sum_{k=2}^{K} \frac{(-1)^{k}}{k !} \chi_{k} \Sigma_{f}(\vec{r}, E, t)\left[\iint F\left(E^{\prime}\right) \tilde{G}\left(R, z, t_{f} ; \vec{r}, E^{\prime}, \vec{\Omega}^{\prime}, t\right) \frac{d \vec{\Omega}^{\prime}}{4 \pi} d E^{\prime}\right]^{k}, t \leq t_{f},
\end{aligned}
$$

where $\Sigma_{f}(\vec{r}, E, t)$ and $\Sigma_{t}(\vec{r}, E, t)$ are the macroscopic fission and total cross sections, respectively, $\bar{\nu}(\vec{r}, E, t)$ is the average number of neutrons per fission, $F(E)$ is the spectrum of fission neutrons (delayed neutrons are ignored in this work), $\Sigma_{s}\left(\vec{r}, E \rightarrow E^{\prime}, \vec{\Omega} \cdot \overrightarrow{\Omega^{\prime}}, t\right)$ is the differential scattering cross section, and $\chi_{k}$ are the factorial moments defined as:

$$
\chi_{k}=\sum_{i=k}^{K} i(i-1)(i-2) \ldots(i-k+1) p_{i},
$$

where $p_{i}$ is the fission neutron multiplicity, i.e., the probability that $i$ neutrons are produced in fission. The generating function satisfies the following free surface boundary condition and final time condition:

$$
\begin{aligned}
& \tilde{G}\left(R, z, t_{f} ; \vec{r}, E, \vec{\Omega}, t_{f}\right)=(1-z) \mathcal{I}_{R}(\vec{r}, E, \vec{\Omega}), \\
& \tilde{G}\left(R, z, t_{f} ; \vec{r}, E, \vec{\Omega}, t\right)=0 \quad \vec{r} \in \partial V \quad \vec{e} \cdot \vec{\Omega}>0 \quad t<t_{f},
\end{aligned}
$$

where $\vec{e}$ is the outward facing normal at a point $\vec{r}$ on the bounding surface $\partial V$ and $\mathcal{I}_{R}(\vec{r}, E, \vec{\Omega})$ is the indicator function, defined as

$$
\mathcal{I}_{R}(\vec{r}, E, \vec{\Omega})= \begin{cases}1, & (\vec{r}, E, \vec{\Omega}) \in R \\ 0, & \text { otherwise }\end{cases}
$$

For expediency of the numerical work and without loss of generality, we assume that scattering is isotropic and also that physical properties of the medium are independent of time. The latter condition ensures that the solution is invariant under temporal translations, i.e., the solution depends only on the difference between the final and initial times, $s=t_{f}-t \geq 0$. Expressing Eq. (3) in terms of this now forward time variable, we obtain: 


$$
\begin{aligned}
& \frac{1}{v} \frac{\partial \tilde{G}(R, z ; \vec{r}, E, \vec{\Omega}, s)}{\partial s}-\vec{\Omega} \cdot \nabla \tilde{G}(R, z ; \vec{r}, E, \vec{\Omega}, s)+\Sigma_{t}(\vec{r}, E) \tilde{G}(R, z ; \vec{r}, E, \vec{\Omega}, s)= \\
& \iint \Sigma_{s}\left(\vec{r}, E \rightarrow E^{\prime}\right) \tilde{G}\left(R, z ; \vec{r}, E^{\prime}, \vec{\Omega}^{\prime}, s\right) \frac{d \vec{\Omega}^{\prime}}{4 \pi} d E^{\prime} \\
& +\bar{\nu}(\vec{r}, E) \Sigma_{f}(\vec{r}, E) \iint F\left(E^{\prime}\right) \tilde{G}\left(R, z ; \vec{r}, E^{\prime}, \vec{\Omega}^{\prime}, s\right) \frac{d \vec{\Omega}^{\prime}}{4 \pi} d E^{\prime} \\
& -\sum_{k=2}^{K} \frac{(-1)^{k}}{k !} \chi_{k} \Sigma_{f}(\vec{r}, E)\left[\iint F\left(E^{\prime}\right) \tilde{G}\left(R, z ; \vec{r}, E^{\prime}, \vec{\Omega}^{\prime}, s\right) \frac{d \vec{\Omega}^{\prime}}{4 \pi} d E^{\prime}\right]^{k}, s \geq 0
\end{aligned}
$$

with boundary and, now, initial conditions given by:

$$
\begin{aligned}
& \tilde{G}(R, z ; \vec{r}, E, \vec{\Omega}, 0)=(1-z) \mathcal{I}_{R}(\vec{r}, \vec{\Omega}) \\
& \tilde{G}(R, z ; \vec{r}, E, \vec{\Omega}, s)=0 \quad \vec{r} \in \partial V \quad \vec{e} \cdot \vec{\Omega}>0 \quad s>0
\end{aligned}
$$

\section{II.A. Incorporating an Intrinsic Random Source}

In order to account for an intrinsic random source, such as spontaneous fission that is of interest here, it is necessary to utilise an auxiliary equation that depends on the neutron distribution due to individual neutron chains, i.e., $p_{n}\left(R, t_{f} ; \vec{r}, E, \vec{\Omega}, t\right)$. To this end, we define $\theta_{n}\left(t_{f} \mid t\right)$ as the probability of finding $n$ neutrons at time $t_{f}$ given that a neutron source was first "turned on" at an earlier time $t \leq t_{f}$. That is, there are no neutrons in the system prior to this time. The source of strength $Q(t)$ is assumed to emit isotropically and is distributed in phase space as $w(\vec{r}, E) / 4 \pi$ with the normalisation $\iiint w(\vec{r}, E) d V d E d \vec{\Omega}=1$. The source multiplicity is given by $q_{k}, k=1,2, \ldots, K_{Q}$ and is assumed normalised to unity. We further assume that the source energy spectrum is invariant in the region where the source is extant and consequently express $w(\vec{r}, E)=w(\vec{r}) F_{Q}(E)$. The source generating function, $G^{(Q)}$, defined as

$$
G^{(Q)}\left(z, t_{f} ; t\right)=\sum_{n=0}^{\infty} z^{n} \theta_{n}\left(t_{f} ; t\right),
$$

can be shown to satisfy the differential equation $[12,9]$ :

$$
\begin{aligned}
& \frac{\partial G^{(Q)}\left(z, t_{f} ; t\right)}{\partial t}=Q(t) \times \\
& \left\{1-\sum_{k=1}^{K_{Q}} q_{k} \int_{V} w(\vec{r})\left[\iint \frac{1}{4 \pi} F_{Q}(E) G\left(R, z, t_{f} ; \vec{r}, E, \vec{\Omega}, t\right) d \vec{\Omega} d E\right]^{k} d V\right\} G^{(Q)}\left(z, t_{f} ; t\right),
\end{aligned}
$$

and is subject to the final condition ,

$$
G^{(Q)}\left(z, t_{f} ; t_{f}\right)=1
$$


corresponding to certainty of no neutrons existing prior to the source being turned on. Defining the factorial moments associated with the source multiplicity as

$$
\chi_{j}^{(Q)}=\sum_{k=j}^{K_{Q}} k(k-1)(k-2) \ldots(k-j+1) q_{k} .
$$

Eq. (10) can be rewritten in terms of the modified generating function for the single initial neutron, $\tilde{G}\left(R, z, t_{f} ; \vec{r}, E, \vec{\Omega}, t\right)$, as,

$$
\begin{aligned}
& \frac{\partial G^{(Q)}\left(z, t_{f} ; t\right)}{\partial t}=-Q(t) \times \\
& {\left[\sum_{k=1}^{K_{Q}} \frac{(-1)^{k}}{k !} \chi_{k}^{(Q)} \int_{V} w(\vec{r})\left[\iint F_{Q}\left(E^{\prime}\right) \tilde{G}\left(R, z, t_{f} ; \vec{r}, E^{\prime}, \vec{\Omega}^{\prime}, t\right) \frac{d \vec{\Omega}^{\prime}}{4 \pi} d E^{\prime}\right]^{k}\right]_{t \leq t_{f}}^{(Q)}\left(z, t_{f} ; t\right) .}
\end{aligned}
$$

Again, assuming time-invariant material properties and source strength, the solution depends on only the time difference $s=t_{f}-t$, allowing us to write Eq. (13) as,

$$
\begin{aligned}
& \frac{\partial G^{(Q)}(z ; s)}{\partial s}= \\
& Q\left[\sum_{k=1}^{K_{Q}} \frac{(-1)^{k}}{k !} \chi_{k}^{(Q)} \int_{V} w(\vec{r})\left[\iint_{Q}\left(E^{\prime}\right) \tilde{G}\left(R, z ; \vec{r}, E^{\prime}, \vec{\Omega}^{\prime}, s\right) \frac{d \vec{\Omega}^{\prime}}{4 \pi} d E^{\prime}\right]^{k}\right] \\
& s \geq 0 \\
& G^{(Q)}(z ; s),
\end{aligned}
$$

with the initial condition:

$$
G^{(Q)}(z ; 0)=1
$$

\section{Equations for Individual Number Probabilities}

We now develop equations for the individual number probabilities $p_{n}(\vec{r}, E, \vec{\Omega}, s)$ (we suppress dependence on the final state subvolume for notational simplicity) from the equations for the generating functions for the single initiating neutron and intrinsic source that were presented above. Numerical solution of these equations will be taken up in the next section for a specific problem geometry.

\section{III.A. Single Initiating Neutron}

Extracting individual number probabilities from the generating function itself can be easily achieved through the evaluation of Eqs. (1) and (2) and their derivatives

with respect to $z$ at $z=0$, giving in the case of $\tilde{G}$ 


$$
\begin{array}{r}
p_{s}(\vec{r}, E, \vec{\Omega}, s)=\tilde{G}(R, z=0 ; \vec{r}, E, \vec{\Omega}, s) \\
p_{n}(\vec{r}, E, \vec{\Omega}, s)=-\left.\frac{1}{n !} \frac{\partial^{n} \tilde{G}(R, z ; \vec{r}, E, \vec{\Omega}, s)}{\partial z^{n}}\right|_{z=0}
\end{array}
$$

where $p_{s}(\vec{r}, E, \vec{\Omega}, s)=1-p_{0}(\vec{r}, E, \vec{\Omega}, s)$ is the survival probability and we note for completeness that since the number distribution $p_{n}(\vec{r}, E, \vec{\Omega}, s), n=0,1, \cdots$, are normalised, it follows that,

$$
G(R, z=1 ; \vec{r}, E, \vec{\Omega}, s)=\sum_{n=0}^{\infty} p_{n}(\vec{r}, E, \Omega, s)=1 .
$$

As $z$ does not appear explicitly in Eq. (7), equations for the individual probabilities can be established by differentiating Eq. (7) and then setting $z=0$ as shown in Eq. (16). We explicitly illustrate the process for the first few equations below.

The equation for the survival probability can be obtained directly through the evaluation of Eq. (7) at $z=0$, as

$$
\begin{aligned}
& \frac{1}{v} \frac{\partial p_{s}(\vec{r}, E, \vec{\Omega}, s)}{\partial s}-\vec{\Omega} . \nabla p_{s}(\vec{r}, E, \vec{\Omega}, s)+\Sigma_{t}(\vec{r}, E) p_{s}(\vec{r}, E, \vec{\Omega}, s)= \\
& \iint \Sigma_{s}\left(\vec{r}, E \rightarrow E^{\prime}\right) p_{s}\left(\vec{r}, E^{\prime}, \vec{\Omega}^{\prime}, s\right) \frac{d \vec{\Omega}^{\prime}}{4 \pi} d E^{\prime} \\
& +\bar{\nu}(\vec{r}, E) \Sigma_{f}(\vec{r}, E) \iint F\left(E^{\prime}\right) p_{s}\left(\vec{r}, E^{\prime}, \vec{\Omega}^{\prime}, s\right) \frac{d \vec{\Omega}^{\prime}}{4 \pi} d E^{\prime} \\
& -\sum_{k=2}^{K} \frac{(-1)^{k}}{k !} \chi_{k} \Sigma_{f}(\vec{r}, E)\left[\iint F\left(E^{\prime}\right) p_{s}\left(\vec{r}, E^{\prime}, \vec{\Omega}^{\prime}, s\right) \frac{d \vec{\Omega}^{\prime}}{4 \pi} d E^{\prime}\right]^{k}
\end{aligned}
$$

If instead we differentiate Eq. (7) and set $z=0$ we obtain the equation for $p_{1}(\vec{r}, E, \vec{\Omega}, s)$

$$
\begin{aligned}
& \frac{1}{v} \frac{\partial p_{1}(\vec{r}, E, \vec{\Omega}, s)}{\partial s}-\vec{\Omega} \cdot \nabla p_{1}(\vec{r}, E, \vec{\Omega}, s)+\Sigma_{t}(\vec{r}, E) p_{1}(\vec{r}, E, \vec{\Omega}, s)= \\
& \iint \Sigma_{s}\left(\vec{r}, E \rightarrow E^{\prime}\right) p_{1}\left(\vec{r}, E^{\prime}, \vec{\Omega}^{\prime}, s\right) \frac{d \vec{\Omega}^{\prime}}{4 \pi} d E^{\prime} \\
& +\bar{\nu}(\vec{r}, E) \Sigma_{f}(\vec{r}, E) \iint F\left(E^{\prime}\right) p_{1}\left(\vec{r}, E^{\prime}, \vec{\Omega}^{\prime}, s\right) \frac{d \vec{\Omega}^{\prime}}{4 \pi} d E^{\prime} \\
& +\sum_{k=2}^{K} \frac{(-1)^{k}}{(k-1) !} \chi_{k} \Sigma_{f}(\vec{r}, E)\left[\iint F\left(E^{\prime}\right) p_{s}\left(\vec{r}, E^{\prime}, \vec{\Omega}^{\prime}, s\right) \frac{d \vec{\Omega}^{\prime}}{4 \pi} d E^{\prime}\right]^{k-1} \\
& \times\left[\iint F\left(E^{\prime}\right) p_{1}\left(\vec{r}, E^{\prime}, \vec{\Omega}^{\prime}, s\right) \frac{d \vec{\Omega}^{\prime}}{4 \pi} d E^{\prime}\right]
\end{aligned}
$$


following the same procedure for $p_{2}(\vec{r}, E, \vec{\Omega}, s)$, gives

$$
\begin{aligned}
& \frac{1}{v} \frac{\partial p_{2}(\vec{r}, E, \vec{\Omega}, s)}{\partial s}-\vec{\Omega} . \nabla p_{2}(\vec{r}, E, \vec{\Omega}, s)+\Sigma_{t}(\vec{r}, E) p_{2}(\vec{r}, E, \vec{\Omega}, s)= \\
& \iint \Sigma_{s}\left(\vec{r}, E \rightarrow E^{\prime}\right) p_{2}\left(\vec{r}, E^{\prime}, \vec{\Omega}^{\prime}, s\right) \frac{d \vec{\Omega}^{\prime}}{4 \pi} d E^{\prime} \\
& +\bar{\nu}(\vec{r}, E) \Sigma_{f}(\vec{r}, E) \iint F\left(E^{\prime}\right) p_{2}\left(\vec{r}, E^{\prime}, \vec{\Omega}^{\prime}, s\right) \frac{d \vec{\Omega}^{\prime}}{4 \pi} d E^{\prime} \\
& +\frac{1}{2 !} \sum_{k=2}^{K} \frac{(-1)^{k}}{(k-2) !} \chi_{k} \Sigma_{f}(\vec{r}, E)\left[\iint F\left(E^{\prime}\right) p_{s}\left(\vec{r}, E^{\prime}, \vec{\Omega}^{\prime}, s\right) \frac{d \vec{\Omega}^{\prime}}{4 \pi} d E^{\prime}\right]^{k-2} \\
& \times\left[\iint F\left(E^{\prime}\right) p_{1}\left(\vec{r}, E^{\prime}, \vec{\Omega}^{\prime}, s\right) \frac{d \vec{\Omega}^{\prime}}{4 \pi} d E^{\prime}\right]^{2} \\
& -\sum_{k=2}^{K} \frac{(-1)^{k}}{(k-1) !} \chi_{k} \Sigma_{f}(\vec{r}, E)\left[\int F^{\prime} F\left(E^{\prime}\right) p_{s}\left(\vec{r}, E^{\prime}, \vec{\Omega}^{\prime}, s\right) \frac{d \vec{\Omega}^{\prime}}{4 \pi} d E^{\prime}\right]^{k-1} \\
& \times\left[\iint F\left(E^{\prime}\right) p_{2}\left(\vec{r}, E^{\prime}, \vec{\Omega}^{\prime}, s\right) \frac{d \vec{\Omega}^{\prime}}{4 \pi} d E^{\prime}\right]
\end{aligned}
$$

and so on. Eqs. (18), (19), and (20) are subject to the initial conditions

$$
\begin{aligned}
& p_{s}(\vec{r}, E, \vec{\Omega}, 0)=\mathcal{I}_{R}(\vec{r}, E, \vec{\Omega}) \\
& p_{n}(\vec{r}, E, \vec{\Omega}, 0)=\delta_{n, 1} \mathcal{I}_{R}(\vec{r}, E, \vec{\Omega}) \quad n=1,2, \ldots
\end{aligned}
$$

and additionally the boundary condition

$$
\begin{array}{lll}
p_{s}(\vec{r}, E, \vec{\Omega}, s)=0 & \vec{r} \in \partial V & \vec{e} \cdot \vec{\Omega} \geq 0 \\
p_{n}(\vec{r}, E, \vec{\Omega}, s)=0 & \vec{r} \in \partial V & \vec{e} \cdot \vec{\Omega} \geq 0
\end{array}
$$

As can be seen from the equations above, extracting the equations for the number probabilities in this way yields a series of linear coupled differential equations, where the equation for $p_{n}(\vec{r}, E, \vec{\Omega}, s)$ depends on $p_{m}(\vec{r}, E, \vec{\Omega}, s)$ where $m<n$. The exception to this is Eq. (18) for the survival probability which is homogeneous, but nonlinear.

Eqs. (19), (20) and so on have the form of a nonhomogeneous adjoint transport equation (except for time which is forward) with a source term constructed from products of the lower order probabilities. It is the calculation of this source term which provides significant complication to the solution of this set of equations. In previous work $[2,13]$ these were calculated using symbolic algebra codes. However, we have developed our own algorithm for calculating these, details of which can be found in the appendix at the end of this paper

\section{III.B. Intrinsic Source}

In a similar manner to the single initiating neutron case, the extraction of the source number probabilities $\theta_{n}(s)$ can be achieved through the evaluation of Eq. (9) 
and its derivatives at $z=0$ as

$$
\begin{aligned}
& G^{(Q)}(z=0 ; \vec{r}, s)=\theta_{0}(\vec{r}, s) \\
& \left.\frac{1}{n !} \frac{\partial^{n} G^{(Q)}(z ; \vec{r}, s)}{\partial z^{n}}\right|_{z=0}=\theta_{n}(\vec{r}, s)
\end{aligned}
$$

Again, as $z$ does not appear explicitly in Eq. (14), to establish the equations for solution simply requires the differentiation of Eq. (14) and then its evaluation at $z=0$. Demonstrating this for Eq. (14), gives for $\theta_{0}$

$$
\frac{\partial \theta_{0}(s)}{\partial s}=Q(\vec{r})\left[\sum_{k=1}^{K_{Q}} \frac{(-1)^{k}}{k !} \chi_{k}^{(Q)} \int_{V} w(\vec{r})\left[\iint F_{Q}\left(E^{\prime}\right) p_{s}\left(\vec{r}, E^{\prime}, \vec{\Omega}^{\prime}, s\right) \frac{d \vec{\Omega}^{\prime}}{4 \pi} d E^{\prime}\right]^{k}\right] \theta_{0}(s)
$$

with the initial condition

$$
\theta_{0}(0)=1
$$

If we instead differentiate Eq. (14) and then set $z=0$, we obtain for $\theta_{1}$

$$
\begin{aligned}
& \frac{\partial \theta_{1}(s)}{\partial s}=Q(\vec{r})\left[\sum_{k=1}^{K_{Q}} \frac{(-1)^{k}}{k !} \chi_{k}^{(Q)} \int_{V} w(\vec{r})\left[\iint F_{Q}\left(E^{\prime}\right) p_{s}\left(\vec{r}, E^{\prime}, \vec{\Omega}^{\prime}, s\right) \frac{d \vec{\Omega}^{\prime}}{4 \pi} d E^{\prime}\right]^{k}\right] \theta_{1}(s) \\
& -Q(\vec{r}) \sum_{k=1}^{K_{Q}} \frac{(-1)^{k}}{(k-1) !} \chi_{k}^{(Q)} \int_{V} w(\vec{r})\left[\iint F_{Q}\left(E^{\prime}\right) p_{s}\left(\vec{r}, E^{\prime}, \vec{\Omega}^{\prime}, s\right) \frac{d \vec{\Omega}^{\prime}}{4 \pi} d E^{\prime}\right]^{k-1} \\
& \times\left[\iint F_{Q}\left(E^{\prime}\right) p_{1}\left(\vec{r}, E^{\prime}, \vec{\Omega}^{\prime}, s\right) \frac{d \vec{\Omega}^{\prime}}{4 \pi} d E^{\prime}\right] \theta_{0}(s)
\end{aligned}
$$

while taking the second derivative and setting $z=0$, gives for $\theta_{2}$ 


$$
\begin{aligned}
& \frac{\partial \theta_{2}(s)}{\partial s}=Q(\vec{r})\left[\sum_{k=1}^{K_{Q}} \frac{(-1)^{k}}{k !} \chi_{k}^{(Q)} \int_{V} w(\vec{r})\left[\iint F_{Q}\left(E^{\prime}\right) p_{s}\left(\vec{r}, E^{\prime}, \vec{\Omega}^{\prime}, s\right) \frac{d \vec{\Omega}^{\prime}}{4 \pi} d E^{\prime}\right] \theta_{2}(s)\right. \\
& -Q(\vec{r}) \sum_{k=1}^{K_{Q}} \frac{(-1)^{k}}{(k-1) !} \chi_{k}^{(Q)} \int_{V} w(\vec{r})\left[\iint F_{Q}\left(E^{\prime}\right) p_{s}\left(\vec{r}, E^{\prime}, \vec{\Omega}^{\prime}, s\right) \frac{d \vec{\Omega}^{\prime}}{4 \pi} d E^{\prime}\right]^{k-1} \\
& \times\left[\iint_{Q}\left(E^{\prime}\right) p_{1}\left(\vec{r}, E^{\prime}, \vec{\Omega}^{\prime}, s\right) \frac{d \vec{\Omega}^{\prime}}{4 \pi} d E^{\prime}\right] \theta_{1}(s) \\
& -Q(\vec{r}) \sum_{k=1}^{K_{Q}} \frac{(-1)^{k}}{(k-1) !} \chi_{k}^{(Q)} \int_{V} w(\vec{r})\left[\iint F_{Q}\left(E^{\prime}\right) p_{s}\left(\vec{r}, E^{\prime}, \vec{\Omega}^{\prime}, s\right) \frac{d \vec{\Omega}^{\prime}}{4 \pi} d E^{\prime}\right]^{k-1} \\
& \times\left[\iint F_{Q}\left(E^{\prime}\right) p_{2}\left(\vec{r}, E^{\prime}, \vec{\Omega}^{\prime}, s\right) \frac{d \vec{\Omega}^{\prime}}{4 \pi} d E^{\prime}\right] \theta_{0}(s) \\
& +\frac{1}{2 !} Q(\vec{r}) \sum_{k=1}^{K_{Q}} \frac{(-1)^{k}}{(k-2) !} \chi_{k}^{(Q)} \int w(\vec{r})\left[\int F_{V}\left(E^{\prime}\right) p_{s}\left(\vec{r}, E^{\prime}, \vec{\Omega}^{\prime}, s\right) \frac{d \vec{\Omega}^{\prime}}{4 \pi} d E^{\prime}\right]^{k-2} \\
& \times\left[\iint F_{Q}\left(E^{\prime}\right) p_{1}\left(\vec{r}, E^{\prime}, \vec{\Omega}^{\prime}, s\right) \frac{d \vec{\Omega}^{\prime}}{4 \pi} d E^{\prime}\right]^{2} \theta_{0}(s)
\end{aligned}
$$

and so on. The $\theta_{n}$ satisfy the initial condition

$$
\theta_{n}(0)=0 \quad n \geq 1
$$

\section{Numerical Solution in Spherical Geometry}

We consider the solution of the coupled set of equations shown in Section (III) for the number probabilities in one dimensional, spherically symmetric geometry consisting of a homogeneous, subcritical mixture of $P u^{239}$ and $P u^{240}$ surrounded by a high-density polyethylene reflector. This geometry is representative of an experimental assembly investigated by [1], although the number probabilities computed here refer to neutrons inside the spherical assembly and not those measured by an external detector. The theoretical formulation can in principle be extended to describe the distribution of neutrons entering a detector external to the spherical assembly but this generalisation is deferred to a future investigation.

\section{IV.A. Single Initiating Neutron}

In spherically symmetric geometry, the spatial and directional dependence of the number probabilities reduces to just the radius $r$ and the cosine of the angle between the radius and direction, i.e., $p_{n}(\vec{r}, E, \vec{\Omega}, s)=p_{n}(r, E, \vec{r} \cdot \vec{\Omega} / r, s)=p_{n}(r, E, \mu, s)$. Equation (18) for the survival probability then be expressed in the following spherically symmetric and conservative form: 


$$
\begin{aligned}
& \frac{1}{v} \frac{\partial p_{s}(r, E, \mu, s)}{\partial s}-\frac{\mu}{r^{2}} \frac{\partial}{\partial r}\left[r^{2} p_{s}(r, E, \mu, s)\right]-\frac{1}{r} \frac{\partial}{\partial \mu}\left[\left(1-\mu^{2}\right) p_{s}(r, E, \mu, s)\right] \\
& +\Sigma_{t}(r, E) p_{s}(r, E, \mu, s)=\frac{1}{2} \iint \Sigma_{s}\left(r, E \rightarrow E^{\prime}\right) p_{s}\left(r, E^{\prime}, \mu^{\prime}, s\right) d \mu^{\prime} d E^{\prime} \\
& +\bar{\nu}(r, E) \Sigma_{f}(r, E) \frac{1}{2} \iint F\left(E^{\prime}\right) p_{s}\left(r, E^{\prime}, \mu^{\prime}, s\right) d \mu^{\prime} d E^{\prime} \\
& -\sum_{k=2}^{K} \frac{(-1)^{k}}{k !} \chi_{k} \Sigma_{f}(r, E)\left[\frac{1}{2} \iint F\left(E^{\prime}\right) p_{s}\left(r, E^{\prime}, \mu^{\prime}, s\right) d \mu^{\prime} d E^{\prime}\right]^{k}
\end{aligned}
$$

We now introduce a new variable $\hat{p}_{s}(r, E, \mu, s)=p_{s}(r, E,-\mu, s)$ allowing us to re-write this equation using a forward angular variable (note, the energy dependence is still adjoint), as

$$
\begin{aligned}
& \frac{1}{v} \frac{\partial \hat{p}_{s}(r, E, \mu, s)}{\partial s}+\frac{\mu}{r^{2}} \frac{\partial}{\partial r}\left[r^{2} \hat{p}_{s}(r, E, \mu, s)\right]+\frac{1}{r} \frac{\partial}{\partial \mu}\left[\left(1-\mu^{2}\right) \hat{p}_{s}(r, E, \mu, s)\right] \\
& +\Sigma_{t}(r, E) \hat{p}_{s}(r, E, \mu, s)=\frac{1}{2} \iint \Sigma_{s}\left(r, E \rightarrow E^{\prime}\right) \hat{p}_{s}\left(r, E^{\prime}, \mu^{\prime}, s\right) d \mu^{\prime} d E^{\prime} \\
& +\bar{\nu}(r, E) \Sigma_{f}(r, E) \frac{1}{2} \iint F\left(E^{\prime}\right) \hat{p}_{s}\left(r, E^{\prime}, \mu^{\prime}, s\right) d \mu^{\prime} d E^{\prime} \\
& -\sum_{k=2}^{K} \frac{(-1)^{k}}{k !} \chi_{k} \Sigma_{f}(r, E)\left[\frac{1}{2} \iint F\left(E^{\prime}\right) \hat{p}_{s}\left(r, E^{\prime}, \mu^{\prime}, s\right) d \mu^{\prime} d E^{\prime}\right]^{k}
\end{aligned}
$$

and the boundary condition on the sphere surface at $r=r_{B}$ are rewritten for incoming directions as:

$$
\begin{aligned}
& \hat{p}_{s}\left(r_{B}, E, \mu, s\right)=0, \quad-1<\mu<0 \\
& \hat{p}_{n}\left(r_{B}, E, \mu, s\right)=0, \quad-1<\mu<0, \quad n=1,2, \ldots
\end{aligned}
$$

The desired survival probability is then obtained by simply transposing the discrete ordinates.

The numerical solution of Eq.(30) requires discretisation of the spatial, angular, energy and time variables as well as a prescription for handling the nonlinearity. Here, we invoke the conventional multigroup approximation in energy [14] to write Eq.(30) as: 


$$
\begin{aligned}
& \frac{1}{v} \frac{\partial \hat{p}_{s g}(r, \mu, s)}{\partial s}+\frac{\mu}{r^{2}} \frac{\partial}{\partial r}\left[r^{2} \hat{p}_{s g}(r, \mu, s)\right]+\frac{1}{r} \frac{\partial}{\partial \mu}\left[\left(1-\mu^{2}\right) \hat{p}_{s g}(r, \mu, s)\right] \\
& +\Sigma_{t g}(r) \hat{p}_{s g}(r, \mu, s)=\sum_{g^{\prime}} \Sigma_{s g \rightarrow g^{\prime}}(r) \frac{1}{2} \int \hat{p}_{s g^{\prime}}\left(r, \mu^{\prime}, s\right) d \mu^{\prime} \\
& +\bar{\nu}_{g}(r) \Sigma_{f g}(r) \sum_{g^{\prime}} F_{g^{\prime}} \frac{1}{2} \int \hat{p}_{s g^{\prime}}\left(r, \mu^{\prime}, s\right) d \mu^{\prime} \\
& -\sum_{k=2}^{K} \frac{(-1)^{k}}{k !} \chi_{k} \Sigma_{f g}(r) \sum_{g^{\prime}} F_{g^{\prime}}\left[\frac{1}{2} \int \hat{p}_{s g^{\prime}}\left(r, \mu^{\prime}, s\right) d \mu^{\prime}\right]^{k}
\end{aligned}
$$

where $1 \leq g \leq G$ is the group index such that group 1 corresponds to neutron energies in the highest energy range and group $G$ corresponds to neutron energies in the lowest energy range. The energy ranges associated with the 6-group structure used for numerical work in this paper are shown in Table (II) below.

Next, we use fully implicit differencing in time and Picard or Richardson iteration [15] to converge the nonlinear term. Using the iteration index, $i$ we now discretise Eq. (32) in time, to obtain

$$
\begin{aligned}
& \frac{1}{v} \frac{\hat{p}_{s, g, j+1}^{(i+1)}(r, \mu)-\hat{p}_{s, g, j}^{\infty}(r, \mu)}{\Delta s}+\frac{\mu}{r^{2}} \frac{\partial}{\partial r}\left[r^{2} \hat{p}_{s, g, j+1}^{(i+1)}(r, \mu)\right] \\
& +\frac{1}{r} \frac{\partial}{\partial \mu}\left[\left(1-\mu^{2}\right) \hat{p}_{s, g, j+1}^{(i+1)}(r, \mu)\right]+\Sigma_{t g}(r) \hat{p}_{s, g, j+1}^{(i+1)}(r, \mu)= \\
& \sum_{g^{\prime}} \Sigma_{r, s g \rightarrow g^{\prime}}(r) \frac{1}{2} \int \hat{p}_{s, g^{\prime}, j+1}^{(i+1)}\left(r, \mu^{\prime}\right) d \mu^{\prime} \\
& +\bar{\nu}_{g}(r) \Sigma_{f g}(r) \sum_{g^{\prime}} F_{g^{\prime}} \frac{1}{2} \int \hat{p}_{s, g^{\prime}, j+1}^{(i)}\left(r, \mu^{\prime}\right) d \mu^{\prime} \\
& -\sum_{k=2}^{K} \frac{(-1)^{k}}{k !} \chi_{k} \Sigma_{f g}(r) \sum_{g^{\prime}} F_{g^{\prime}}\left[\frac{1}{2} \int \hat{p}_{s, g^{\prime}, j+1}^{(i)}(r, \mu) d \mu^{\prime}\right]^{k}
\end{aligned}
$$

where $j$ denotes the time discretisation index, $i$ the Picard iteration index and we have used notation such that $\hat{p}_{s, g, i}^{\infty}(r, \mu)$ denotes a converged solution at the previous time step.

Finally, we use discrete ordinates in angle and a weighted diamond difference scheme in spherical geometry in both space and angle [14], but where the angular weighting is adjusted to eliminate the well documented flux dip observed in this method as is done in [16]. As this a standard implementation of the method and is well documented, no further details are provided about the numerical scheme.

As the numerical solution requires the knowledge of the survival probability in all groups and all angles at each iteration of the nonlinear term, nested sweeps were re- 
quired to converge over angle and group solutions at every time step. We chose to iterate such that the inner most sweep solved over space and angle, the next outward sweep solved over the energy groups and the outermost iterated over the non-linearity until convergence was reached at each time step. Whist other authors [17] have demonstrated more elegant solution techniques to deal with the nonlinearity, we found it was sufficient to perform a Picard scheme, with this convergence typically taking eight iterations. The computational efficiency of the Picard iteration in handling the nonlinear terms was separately established within a lumped model setting by comparing against a Newton scheme. It was found that both schemes took a similar number of iterations to converge for the class of problems of interest here but the cost of the computation was slightly higher for the Newton scheme. For this reason as well as ease of implementation, Picard iteration was used in the more general nonlumped model of this paper. The rapid convergence of the iterations is attributed to starting the iteration at any time step with the converged solution from the previous time step and to the fact that the magnitude of the nonlinear term is small for the subcritical conditions investigated here. Finally, whilst the equations for the number probabilities of order greater than or equal to one are linear, we note that convergence still has to occur over the fission and scattering terms, thus the process of solving these equations proceeds in exactly the same manner as demonstrated above but without the Picard iteration step.

We now apply this methodology by solving this set of equations for a system similar to that described in [1]: namely a $3.7938 \mathrm{~cm}$ sphere of a homogeneous mixture of $5.91 \% \mathrm{Pu}^{240}$ and $93.27 \% \mathrm{Pu}^{239}$ with the additional mass made up from other isotopes of $\mathrm{Pu}$, surrounded by a $1 \mathrm{~cm}$ thick reflector made of high-density polyethylene. The induced fission probabilities used were those given in table (1) of [2] which we have reproduced in table (I) for convenience where we note that these multiplicities relate to the system considered in [2], consisting of $80 \%{ }^{239} \mathrm{Pu}$ and $20 \%{ }^{240} \mathrm{Pu}$, in the absence of multiplicity data for the composition considered in this paper. The six group crosssections were created using [18] by reducing the 172 group XMAS group structure into six groups with energy boundaries based on those used by [19] which also provided the group velocities and are given in tables (II) and (III).

The numerical results reported below were obtained for $S_{8}$ angular discretisation, a timestep of $\Delta s=1 \times 10^{-11}$ sec and 190 spatial cells. These results are accurate to within $1 \%$ of the "exact" values as defined by an ultra-refined solution.

\begin{tabular}{|l|ccccccccc|}
\hline Neutrons & 0 & 1 & 2 & 3 & 4 & 5 & 6 & 7 & 8 \\
\hline$p_{f}(n)$ & 0.0071 & 0.0674 & 0.2283 & 0.3263 & 0.2510 & 0.0958 & 0.0208 & 0.0029 & 0.0005 \\
\hline
\end{tabular}

Table I: Induced fission probabilities, reproduced from [2] 


\begin{tabular}{|l|cccccc|}
\hline group & 1 & 2 & 3 & 4 & 5 & 6 \\
\hline $\mathrm{E}(\mathrm{MeV})$ & $3.0-\infty$ & $1.4-3.0$ & $0.9-1.4$ & $0.4-0.9$ & $0.1-0.4$ & $0-0.1$ \\
$\mathrm{v}(\mathrm{cm} / \mathrm{s})$ & $28.5 \times 10^{8}$ & $19.9 \times 10^{8}$ & $14.7 \times 10^{8}$ & $11.0 \times 10^{8}$ & $6.7 \times 10^{8}$ & $2.9 \times 10^{8}$ \\
\hline$F^{g}$ & $2.122 \times 10^{-1}$ & $3.719 \times 10^{-1}$ & $1.475 \times 10^{-1}$ & $1.725 \times 10^{-1}$ & $8.104 \times 10^{-2}$ & $1.488 \times 10^{-2}$ \\
\hline$\Sigma_{t}$ & $3.694 \times 10^{-1}$ & $3.646 \times 10^{-1}$ & $3.528 \times 10^{-1}$ & $4.071 \times 10^{-1}$ & $5.289 \times 10^{-1}$ & 1.102 \\
$\Sigma_{a}$ & $8.494 \times 10^{-2}$ & $9.509 \times 10^{-2}$ & $8.844 \times 10^{-2}$ & $8.099 \times 10^{-2}$ & $7.693 \times 10^{-2}$ & $4.729 \times 10^{-1}$ \\
$\Sigma_{f}$ & $8.541 \times 10^{-2}$ & $9.331 \times 10^{-2}$ & $8.523 \times 10^{-2}$ & $7.584 \times 10^{-2}$ & $6.730 \times 10^{-2}$ & $3.124 \times 10^{-1}$ \\
$\Sigma_{s}^{g \rightarrow 1}$ & $2.236 \times 10^{-1}$ & 0.0 & 0.0 & 0.0 & 0.0 & 0.0 \\
$\sum_{s}^{g \rightarrow 2}$ & $1.281 \times 10^{-2}$ & $2.104 \times 10^{-1}$ & 0.0 & 0.0 & 0.0 & 0.0 \\
$\Sigma_{s}^{g \rightarrow 3}$ & $1.072 \times 10^{-2}$ & $1.310 \times 10^{-2}$ & $2.030 \times 10^{-1}$ & 0.0 & 0.0 & 0.0 \\
$\Sigma_{s}^{g \rightarrow 4}$ & $2.168 \times 10^{-2}$ & $2.556 \times 10^{-2}$ & $3.066 \times 10^{-2}$ & $2.889 \times 10^{-1}$ & 0.0 & 0.0 \\
$\Sigma_{s}^{g \rightarrow 5}$ & $1.364 \times 10^{-2}$ & $1.748 \times 10^{-2}$ & $2.577 \times 10^{-2}$ & $2.935 \times 10^{-2}$ & $4.286 \times 10^{-1}$ & 0.0 \\
$\sum_{s}^{g \rightarrow 6}$ & $2.011 \times 10^{-3}$ & $2.933 \times 10^{-3}$ & $4.903 \times 10^{-3}$ & $7.819 \times 10^{-3}$ & $2.335 \times 10^{-2}$ & $6.296 \times 10^{-1}$ \\
\hline
\end{tabular}

Table II: Cross sections for the multiplying sphere generated from [18] based on the energy discretisation of [19].

\begin{tabular}{|l|cccccc|}
\hline group & 1 & 2 & 3 & 4 & 5 & 6 \\
\hline $\mathrm{E}(\mathrm{MeV})$ & $3.0-\infty$ & $1.4-3.0$ & $0.9-1.4$ & $0.4-0.9$ & $0.1-0.4$ & $0-0.1$ \\
$\mathrm{v}(\mathrm{cm} / \mathrm{s})$ & $28.5 \times 10^{8}$ & $19.9 \times 10^{8}$ & $14.7 \times 10^{8}$ & $11.0 \times 10^{8}$ & $6.7 \times 10^{8}$ & $2.9 \times 10^{8}$ \\
\hline$F^{g}$ & 0.0 & 0.0 & 0.0 & 0.0 & 0.0 & 0.0 \\
\hline$\Sigma_{t}$ & $2.204 \times 10^{-1}$ & $3.149 \times 10^{-1}$ & $4.280 \times 10^{-1}$ & $5.790 \times 10^{-1}$ & $9.131 \times 10^{-1}$ & 3.596 \\
$\Sigma_{a}$ & $2.804 \times 10^{-4}$ & $3.100 \times 10^{-6}$ & $2.881 \times 10^{-6}$ & $3.042 \times 10^{-6}$ & $5.032 \times 10^{-6}$ & $1.902 \times 10^{-2}$ \\
$\Sigma_{f}$ & 0.0 & 0.0 & 0.0 & 0.0 & 0.0 & 0.0 \\
$\Sigma_{s}^{g \rightarrow 1}$ & $8.544 \times 10^{-2}$ & 0.0 & 0.0 & 0.0 & 0.0 & 0.0 \\
$\Sigma_{s}^{g \rightarrow 2}$ & $8.353 \times 10^{-2}$ & $1.294 \times 10^{-1}$ & 0.0 & 0.0 & 0.0 & 0.0 \\
$\Sigma_{s}^{g \rightarrow 3}$ & $1.686 \times 10^{-2}$ & $7.134 \times 10^{-2}$ & $1.187 \times 10^{-1}$ & 0.0 & 0.0 & 0.0 \\
$\Sigma_{s}^{g \rightarrow 4}$ & $1.891 \times 10^{-2}$ & $6.275 \times 10^{-2}$ & $1.869 \times 10^{-1}$ & $2.432 \times 10^{-1}$ & 0.0 & 0.0 \\
$\Sigma_{s}^{g \rightarrow 5}$ & $1.117 \times 10^{-2}$ & $3.739 \times 10^{-2}$ & $8.899 \times 10^{-2}$ & $2.507 \times 10^{-1}$ & $4.763 \times 10^{-1}$ & 0.0 \\
$\Sigma_{s}^{g \rightarrow 6}$ & $4.164 \times 10^{-3}$ & $1.404 \times 10^{-2}$ & $3.340 \times 10^{-2}$ & $8.518 \times 10^{-2}$ & $4.368 \times 10^{-1}$ & 3.577 \\
\hline
\end{tabular}

Table III: Cross sections for the reflector generated from [18] based on the energy discretisation of [19].

We next present numerical results for the neutron number probabilities in various subvolumes, over different spatial regions, angles and energy groups.

\section{IV.A.1. Number Distribution Over the Full Sphere}

We firstly consider the probabilities of obtaining different numbers of neutrons in a region that comprises the full sphere, regardless of the distribution in energy and angle. As the permutation of initial neutron coordinates is large - specifically six groups, eight discrete ordinates and 191 spatial nodes - we choose to illustrate the methodology by displaying results for a neutron injected into the most extreme groups (fast and thermal) and angles (inward and outward directed) at different positions 
along the radius of the sphere to illustrate the differences between these distributions.
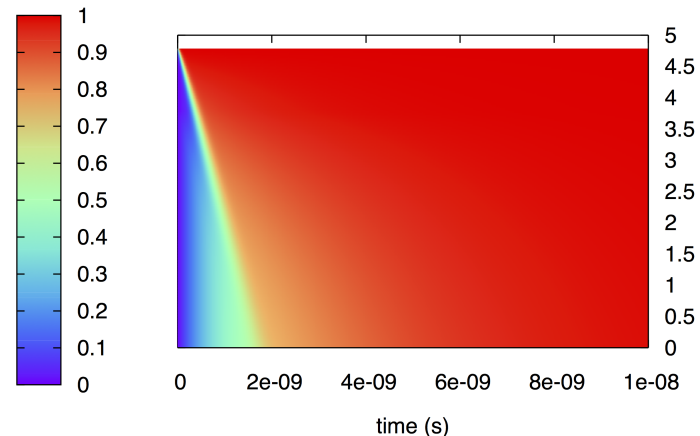

(a) $p_{0}$

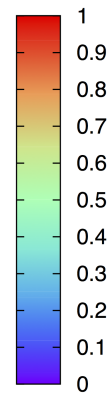

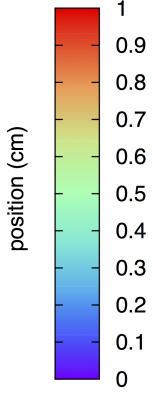

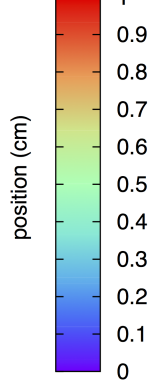

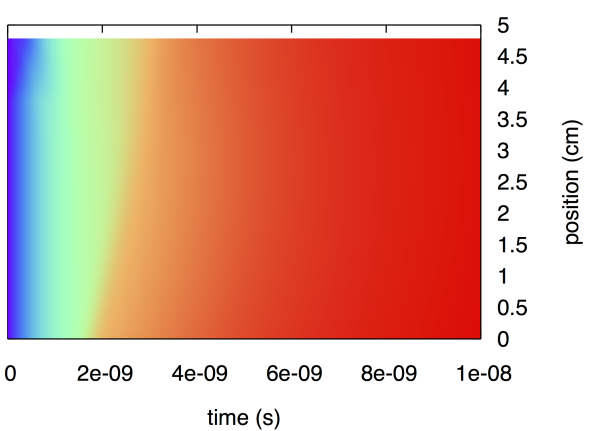

(b) $p_{0}$

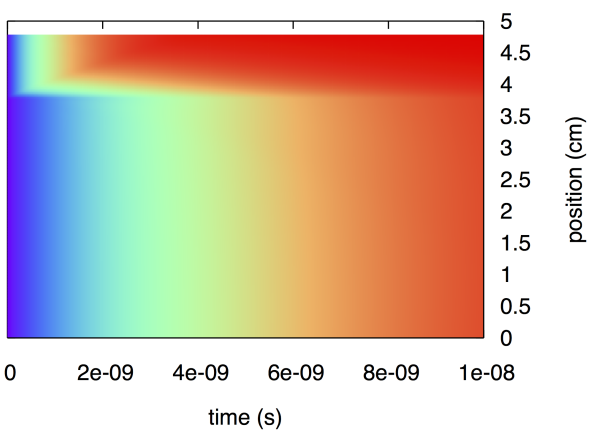

(d) $p_{0}$

(c) $p_{0}$

Figure 1: Probability distribution for finding 0 neutrons in the whole system given that an initial neutron was introduced in (1a) the highest energy group and most outward pointing angle, (1b) the highest energy group and most inward pointing angle, (1c) the lowest energy group and most outward pointing angle and (1d) the lowest energy group and most inward pointing angle.

From the graphs shown in figure (1) it is seen that different angles and energies of injection of the initial neutron make a large difference to the number probability distributions. All figures show the general pattern of starting at zero - as there is one neutron present in the system initially - with the probabilities increasing to one, which is as expected due to the consideration of a subcritical system. However, the inclusion of different energy groups and angles of injection creates a great difference between the spatial and temporal variation of the various distributions.

It is seen that for a neutron injected at a higher energy, figures (1a) and (1b), the process of the extinction of the neutron population in the system happens much more rapidly than for the lower energy neutrons in figures (1c) and (1d). A neutron injected at the higher energy group takes less time to reach the boundary of the system and leak from it, and is also less likely to cause fission events due to the lower fission cross sections shown in table (II). In contrast, a neutron injected at a lower energy is more likely to cause fission events and is more affected by the presence of the reflector, which 
can be easily seen in figures (1c) and (1d).
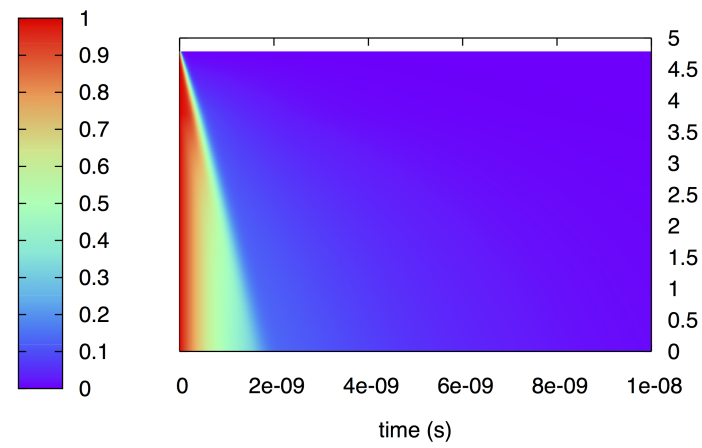

(a) $p_{1}$

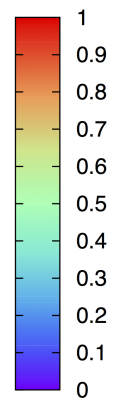

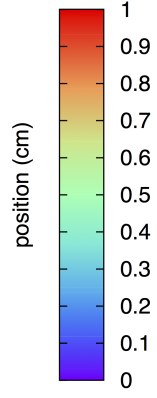

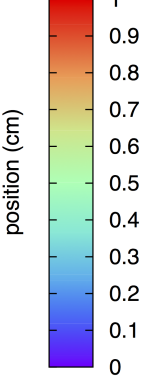

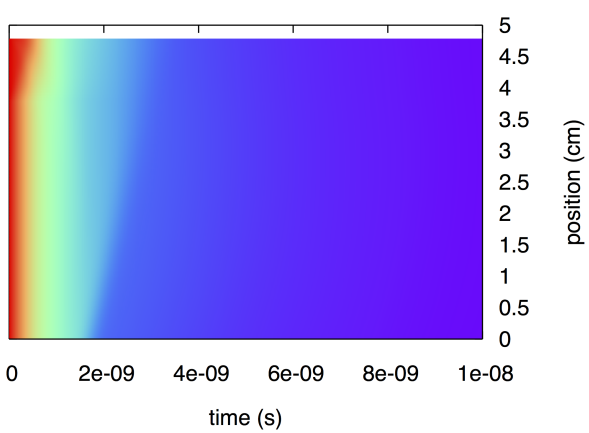

(b) $p_{1}$

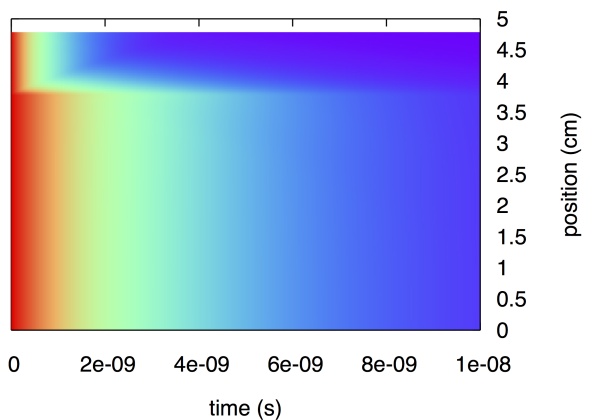

(d) $p_{1}$

(c) $p_{1}$

Figure 2: Probability distribution for finding 1 neutron in the whole system given that an initial neutron was introduced in (2a) the highest energy group and most outward pointing angle, (2b) the highest energy group and most inward pointing angle, (2c) the lowest energy group and most outward pointing angle and (2d) the lowest energy group and most inward pointing angle.

In addition to the energy dependence of the injected neutron making a large difference to the progression of the extinction probability, the angle of the injected neutron is also seen to make a marked difference. If the neutron is injected at an outward pointing angle, (1a) and (1c), the neutron population quickly becomes extinct near the edges of the sphere. If instead it is injected at an inward facing angle, (1b) and (1d), the population is much more long-lived at the boundary. In fact, this is particularly true in the case in which the neutron is injected into the reflector at an inward facing angle. Here, in the case of the highest energy group, the population is seen to be most long-lived when the neutron is injected at the boundary, dropping to a similar temporal progression to an outward facing injection at the centre where the direction will make little difference as the system is spherically symmetric. The high energy group means that neutrons injected in the reflector with an inward facing angle are likely to undergo scattering events, slowing them down and making them more likely to enter the fissile region at lower energies where fission events are more probable: meaning the 
extinction of the population in the system will take longer.

In a broad sense, the graphs in figure (2) shows approximately the opposite behaviour to those for $p_{0}$. The probability starts at one, as one initial neutron is injected, dropping to zero due to the consideration of a sub-critical system. The presence of the reflector is clearly seen in all the figures, except figure (2a), due to the different temporal behaviours observed. In the case of the highest energy group, the lifetime of the neutron is longer when it is injected in the reflector region. This is because scattering collisions in the reflector slow the initial neutron, creating a lower energy neutron which may then migrate to the fissile region where it will a have larger fission cross section than the initial higher energy neutron. In the lowest energy group the reflector is much more likely to absorb the neutrons injected in it than any other group, as demonstrated in table (III), contributing to neutrons injected in this phase space being much less long-lived than their higher energy counterparts.

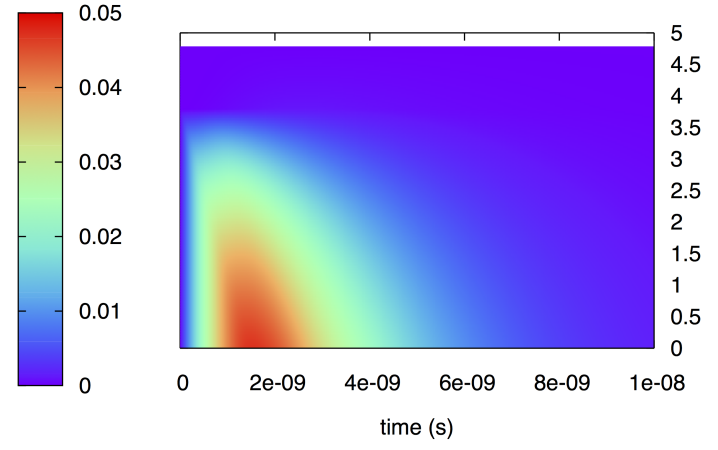

(a) $p_{2}$

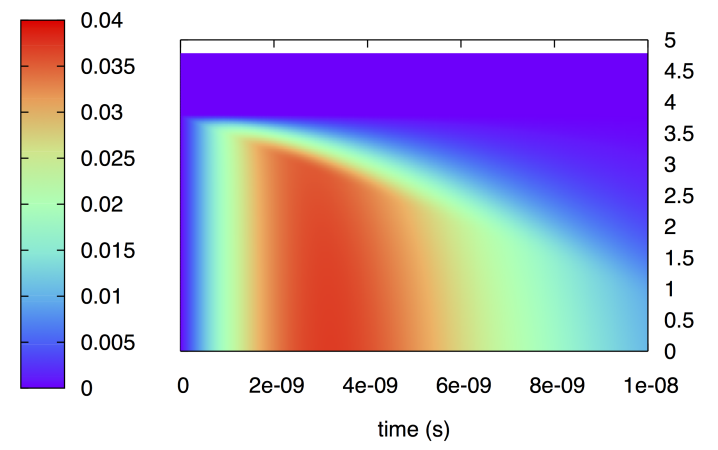

(c) $p_{2}$
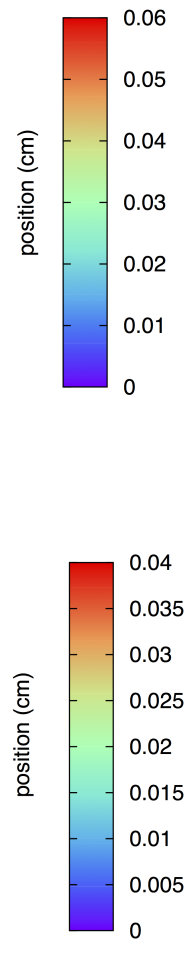

(d) $p_{2}$

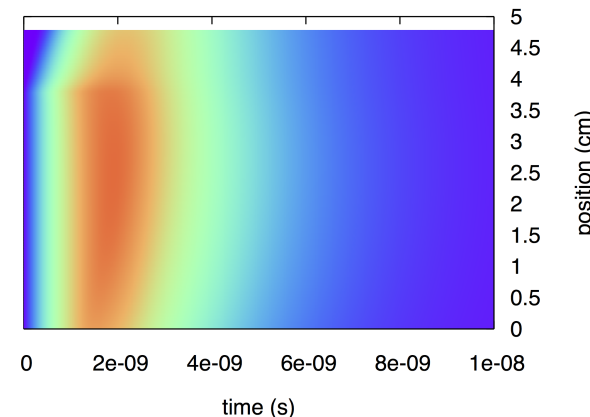

(b) $p_{2}$

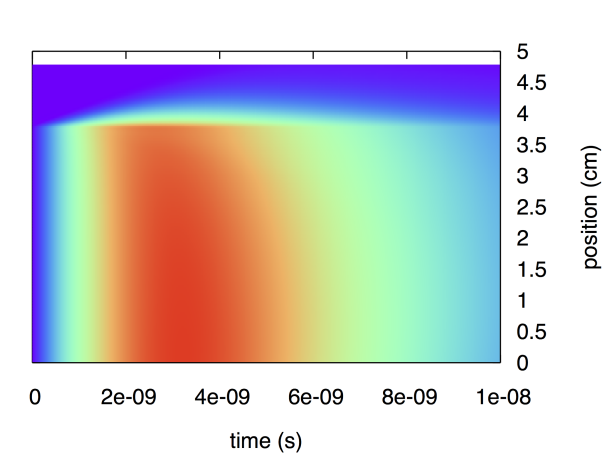
Figure 3: Probability distribution for finding 2 neutrons in the whole system given that
an initial neutron was introduced in (3a) the highest energy group and most outward pointing angle, $(3 \mathrm{~b})$ the highest energy group and most inward pointing angle, $(3 \mathrm{c})$ the lowest energy group and most outward pointing angle and (3d) the lowest energy group and most inward pointing angle.

The probability distributions for $p_{n}$ for $n \geq 2$ follow similar progressions in terms of shape, but differ in magnitude with the higher $n$ having smaller probabilities - as 
is demonstrated by figure (3) when compared to figure (4). We see that the reflector region (the outermost $\mathrm{cm}$ on the $\mathrm{y}$-axis) is most evident in the figures corresponding to when the neutrons have been injected in the low energy group, figures (3c) and (3d), - this is due to the fact that any neutrons injected in this region are more likely to be absorbed than any of the other energy groups and also likely to undergo more scattering events - remaining in the reflector for longer, thus giving them a greater chance of being either absorbed or lost from the system without causing further fissions. Consequently, if the neutron is injected in the reflector region, the probability of finding $n \geq 2$ neutrons in the system is near zero.

Contrasting this, at inward facing injection angles, there is again a significant probability for finding $n \geq 2$ neutrons in the system for injection into the highest energy group.Again, this is because the reflector slows the neutrons to energies where fission is more likely if the neutrons enter the fissile region of the sphere. Finally, we note that the distributions in figure (4) are more strongly peaked in the centre of the sphere than those in figure (3). If a neutron is injected near the centre of the fissile material, it is more likely to undergo fission and less likely to leak making the process of accumulating ten neutrons more likely at these central positions within the sphere.

\section{IV.A.2. Number Distribution Over a Thin Surface Region}

We now change the neutron counting region to cover only the outermost cell in the spatial region, and consider the distribution of neutrons directed out of the sphere regardless of energy. That is, we consider the phase space region defined by

$r_{B}-\delta r \leq r \leq r_{B}, \quad 0<\mu \leq 1, \quad 1 \leq g \leq G$, where $r_{B}$ is the radius of the entire system, $\delta r$ corresponds to the width of the outermost spatial cell and $g$ indicates the group number where $G$ is the total number of energy groups.

The boundary condition imposed is evident in figures (5a) and (5c) showing the sharp dip to zero at the edge of the sphere, corresponding to when the initial neutron is injected in the counting region. Both figures (5a) and (5c) show a shadow protruding diagonally from the corner, corresponding to neutrons which have made their way over to the counting region with relatively few collisions. Note the different angles of the shadows in figures (5a) and (5c) corresponding to the different speeds the neutrons in the two different energy groups travel at.

Figures (5b) and (5d) show a more complex set of behaviours as these are for neutrons injected at the most inward facing angles - we note three individual behaviours. Firstly at the earliest times there is a slight shadow from the edge cell at an angle similar to those seen in figures (5a) and (5c) respectively. This is likely to be due to where neutrons have made their way over to the counting region undergoing very few, perhaps only a single collision, to change their direction - the large scattering cross section of the reflector means that the neutrons are capable of changing direction to be counted. The second behaviour seen is the stronger shadow from the outer edge of the sphere but later in time. This is thought to be due to neutrons which undergo numerous scattering events before making their way to the counting region - the broadness of the feature indicating the various numbers of scattering events which may occur for a 


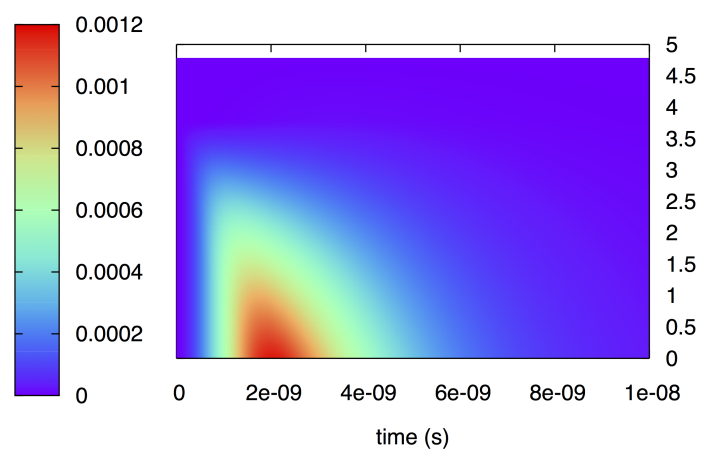

(a) $p_{10}$
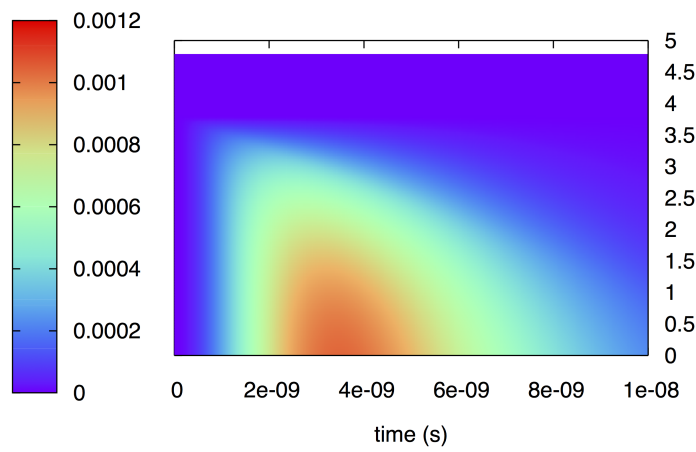

(c) $p_{10}$
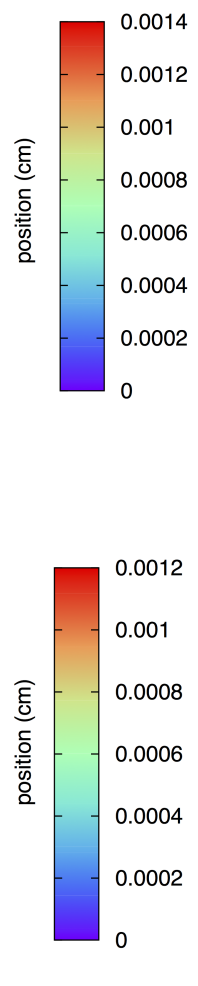

(b) $p_{10}$

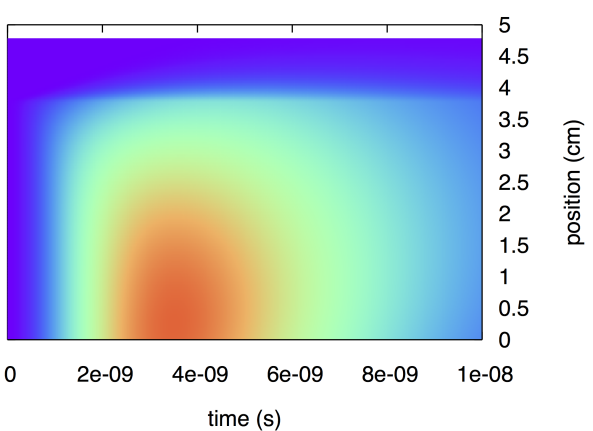

(d) $p_{10}$

Figure 4: Probability distribution for finding 10 neutrons in the whole system given that an initial neutron was introduced in (4a) the highest energy group and most outward pointing angle, (4b) the highest energy group and most inward pointing angle, (4c) the lowest energy group and most outward pointing angle and (4d) the lowest energy group and most inward pointing angle.

neutron to obtain a suitable final direction to be counted. The shadow is observed to be much stronger and more spread out in time in (5b) as compared to (5d) due to the fact that neutrons are more likely to be absorbed by the reflector if they are in the lower energy group.

Finally, a third feature stretches across the whole radius in figure (5b) and the multiplying sphere portion in $(5 \mathrm{~d})$ at later times. In figure (5b) this is thought to be due to neutrons which are either injected in, or migrate to the fissile sphere, undergo fissions and then their progeny make their way to the counting region - this is evident from the opposite slope to the other two features as neutrons injected into the reflector region would be delayed as they would have to undergo scattering twice. The third feature in $(5 \mathrm{~d})$ is thought to not stretch across the whole radius due to the greatly increased likelihood of absorption if the neutron travels across the reflector twice. Neutrons injected in the fissile region here are more likely to cause fission events and then it is these fission neutrons which would make their way to the counting region. 


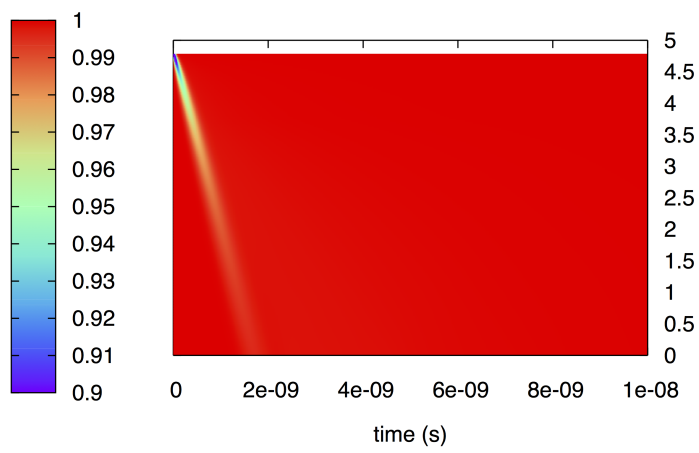

(a) $p_{0}$

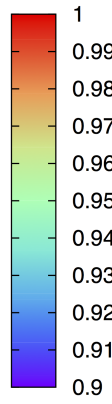

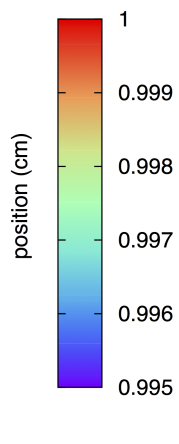

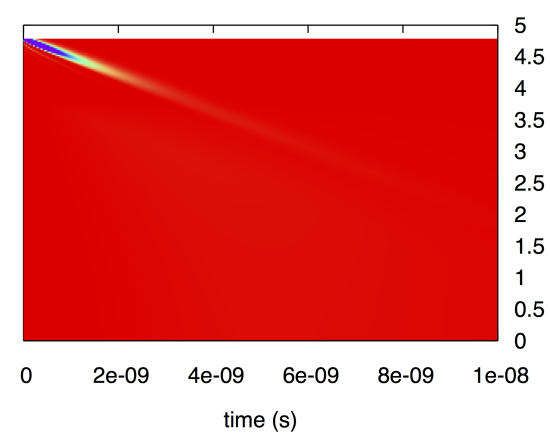

(c) $p_{0}$

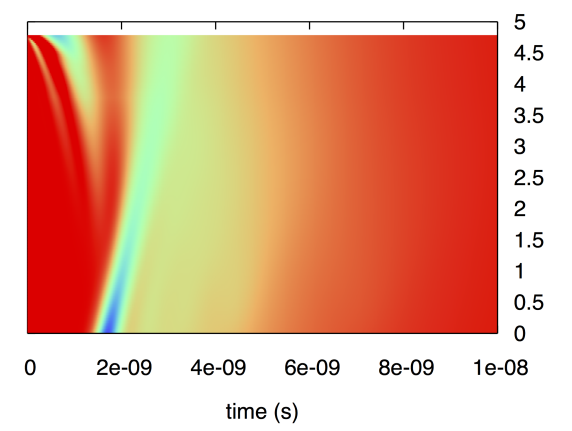

(b) $p_{0}$
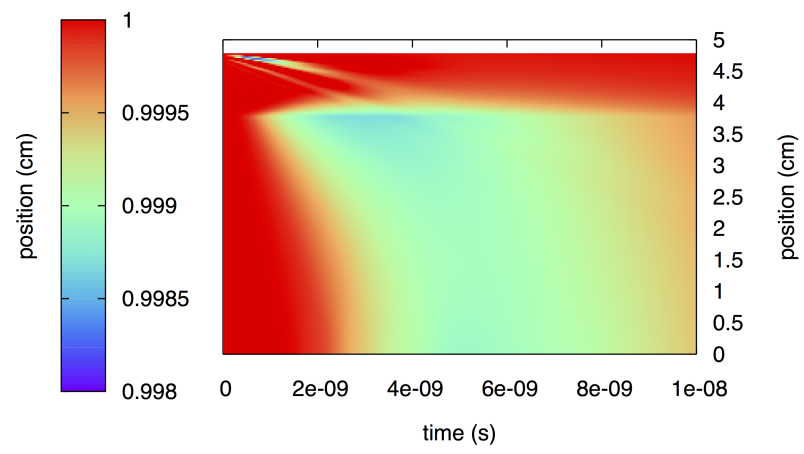

(d) $p_{0}$

Figure 5: Probability distribution for finding 0 neutrons in a thin boundary region given that an initial neutron was introduced in (5a) the highest energy group and most outward pointing angle, (5b) the highest energy group and most inward pointing angle, (5c) the lowest energy group and most outward pointing angle and (5d) the lowest energy group and most inward pointing angle.

We note that approximately the opposite behaviours are seen in the graphs in figure (6) which are explained by the behaviours above.

The graphs in figure (7) all show similar behaviours to the figures for $p_{2}$ in the full sphere case, but with the probabilities being significantly smaller than their full sphere counting region counterparts due to a much smaller counting region. Note also that the features occur later in time than in the full sphere case: this is as, to be counted, the neutrons produced in the fissile region have to migrate across to the counting region.

The distributions for $p_{10}$ were seen to be extremely small in magnitude, being of the order $10^{-23}$ and for that reason are not shown here graphically.

\section{IV.B. Random Intrinsic Source}

We now extend the numerical solutions to account for the presence of a uniformly emitting source throughout the fissile sphere. Taking Eq. (24) and evaluating it in the spherical geometry case, we obtain 


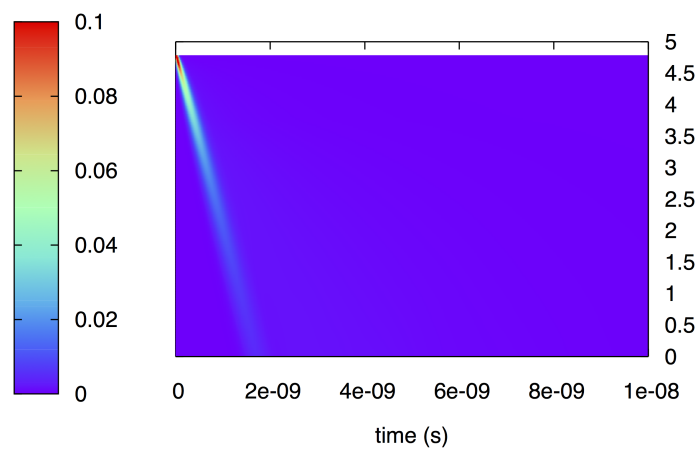

(a) $p_{1}$

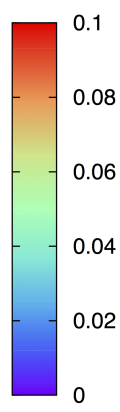

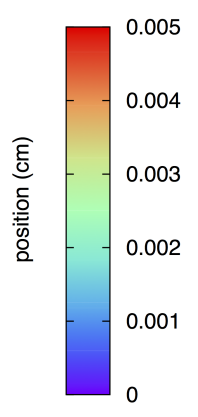$$
0
$$

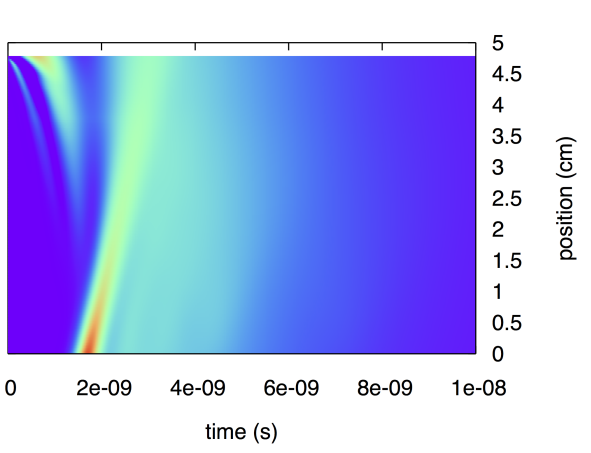

(b) $p_{1}$

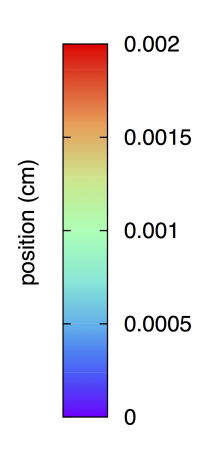

(c) $p_{1}$

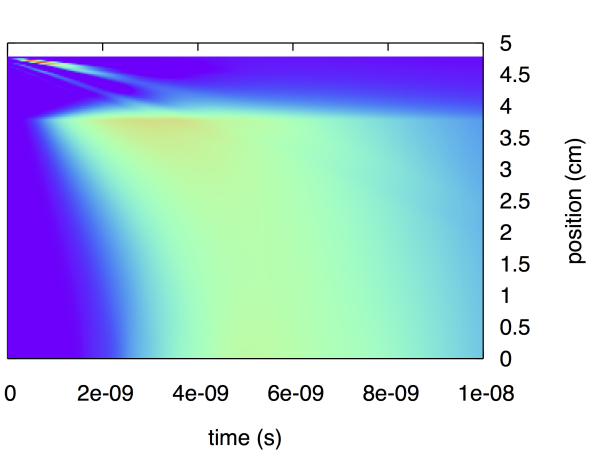

(d) $p_{1}$

Figure 6: Probability distribution for finding 1 neutron in a thin boundary region given that an initial neutron was introduced in (6a) the highest energy group and most outward pointing angle, (6b) the highest energy group and most inward pointing angle, (6c) the lowest energy group and most outward pointing angle and (6d) the lowest energy group and most inward pointing angle.

$\frac{\partial \theta_{0}(s)}{\partial s}=Q\left[\sum_{k=1}^{K_{Q}} \frac{(-1)^{k}}{k !} \chi_{k}^{(Q)} \int_{r=0}^{r_{Q}} 4 \pi r^{2} d r \frac{3}{4 \pi r_{Q}^{3}}\left[\iint F_{Q}\left(E^{\prime}\right) p_{s}\left(\vec{r}, E^{\prime}, \vec{\Omega}^{\prime}, s\right) \frac{d \vec{\Omega}^{\prime}}{4 \pi} d E^{\prime}\right]^{k} \theta_{0}(s)\right.$

where $r_{Q}$ is the radius of the multiplying region, in our case $3.7938 \mathrm{~cm}$, over which the source is assumed to be uniformly distributed, so that $w=1 / V$ and $V=4 \pi r_{Q}^{3} / 3$. Eq. (34) is solved subject to the initial condition

$$
\theta_{0}(0)=1
$$

Eq. (34) was then discretised in space, angle and energy using the same mesh as for the single initial neutron case and solved with a fully implicit time-discretisation scheme.

For the results considered in this section we use the same fission spectrum as in table (II) and a source strength from [1] of $2.78 \times 10^{5} \mathrm{n} / \mathrm{s}$. The factorial moments $\chi_{k}^{(Q)}$ 


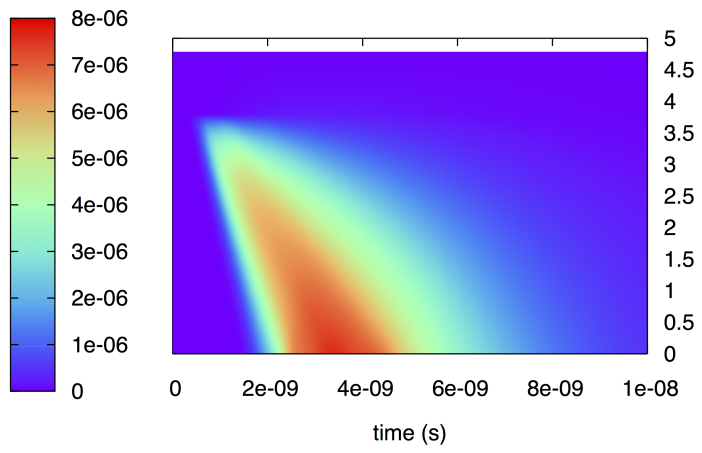

(a) $p_{2}$

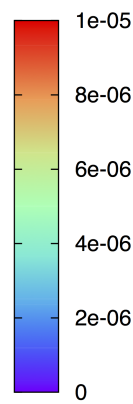

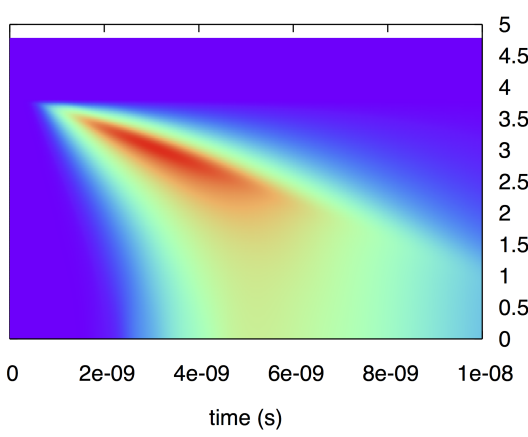
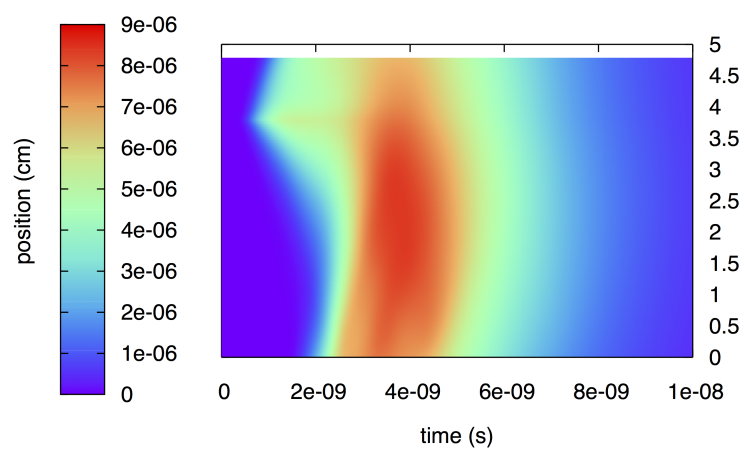

(b) $p_{2}$

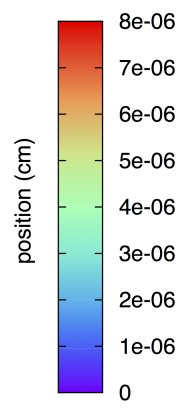

(c) $p_{2}$

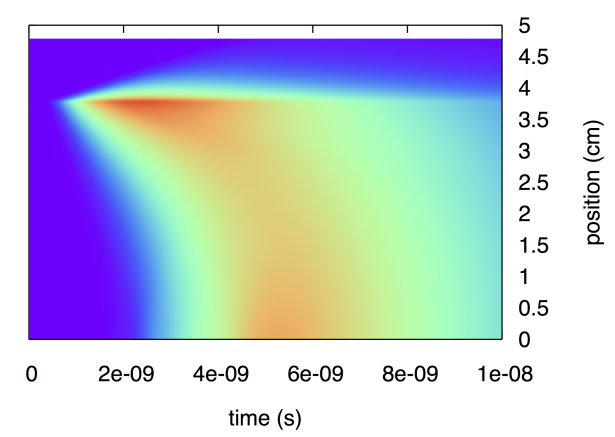

(d) $p_{2}$

Figure 7: Probability distribution for finding 2 neutrons in a thin boundary region given that an initial neutron was introduced in (7a) the highest energy group and most outward pointing angle, (7b) the highest energy group and most inward pointing angle, $(7 \mathrm{c})$ the lowest energy group and most outward pointing angle and $(7 \mathrm{~d})$ the lowest energy group and most inward pointing angle.

were calculated from the spontaneous fission probabilities in [2], which are reproduced in table (IV) for convenience, again these refer to the system consisting of $80 \%{ }^{239} \mathrm{Pu}$ and $20 \%{ }^{240} \mathrm{Pu}$ as considered in that paper.

\begin{tabular}{|l|ccccccc|}
\hline Neutrons & 0 & 1 & 2 & 3 & 4 & 5 & 6 \\
\hline$p_{f}(n)$ & 0.0638 & 0.2316 & 0.3325 & 0.2533 & 0.0987 & 0.0181 & 0.0020 \\
\hline
\end{tabular}

Table IV: Spontaneous fission probabilities, reproduced from [2]

For the higher order $\theta_{n}(s)$, the equations for solution are given in Section (III). These all strongly resemble Eq. (34), but have an additional source term. In this way, they can be dealt with in the same manner as the $\theta_{0}(s)$ case. We now consider results for the various counting regions as considered in the single initiating neutron case.

\section{IV.B.1. Full Sphere Counting Region}

As can be seen from figure (8), finding no neutrons within the entire sphere is the most probable outcome of the assembly. We see that figure (8a) starts at one: as 


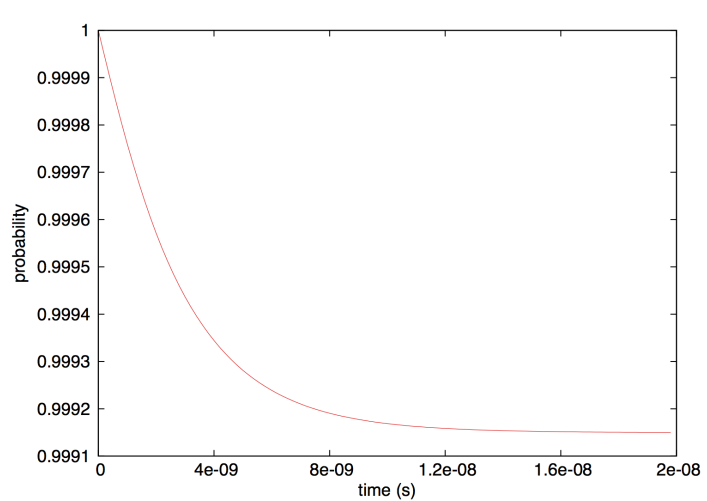

(a) $\theta_{0}$

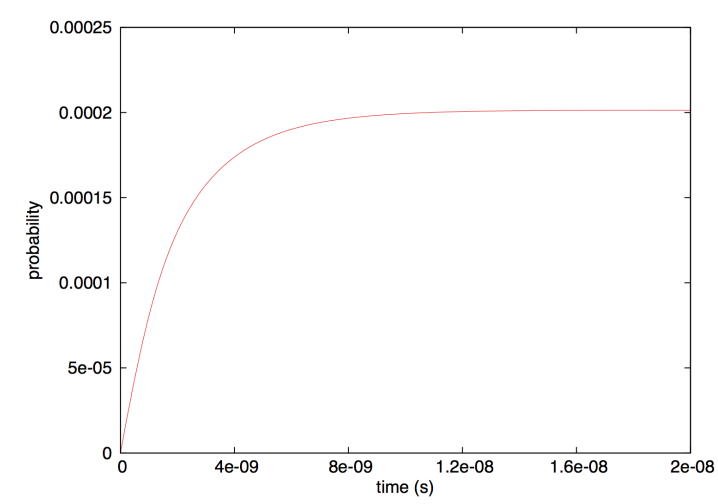

(c) $\theta_{2}$

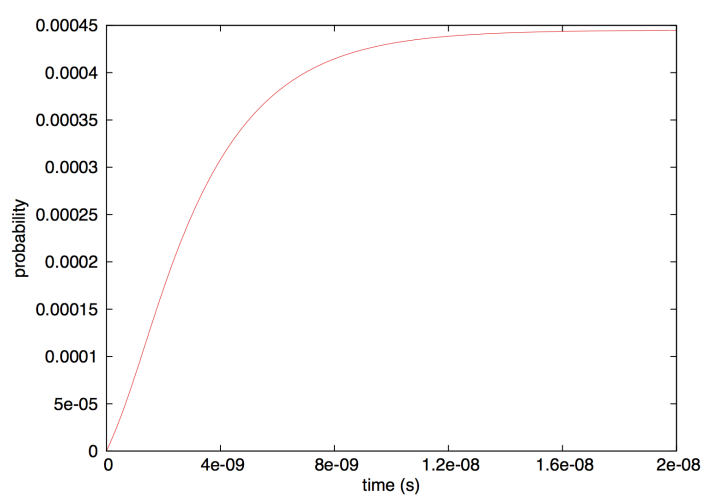

(b) $\theta_{1}$

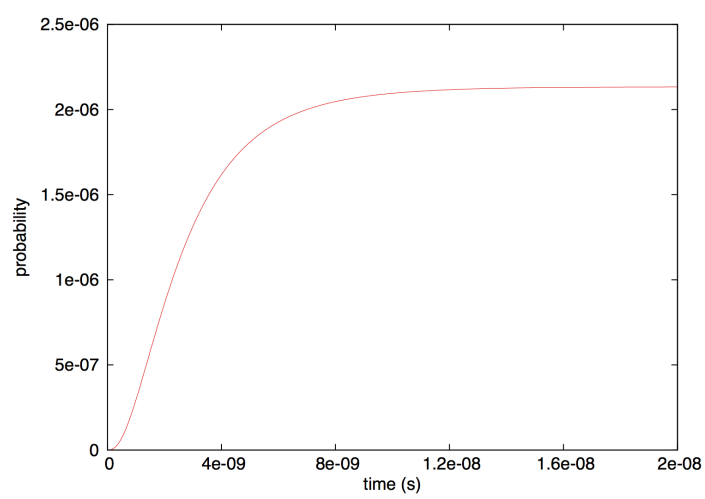

(d) $\theta_{10}$

Figure 8: Probability distribution for finding (8a) zero neutrons, (8b) one neutron, (8c) two neutrons and (8d) ten neutrons in the entire spherical assembly in the presence of a uniform intrinsic source within the sphere.

the initial condition dictates that there are no neutrons in the sphere initially when the source is switched on, this probability then decreases as neutrons are produced in spontaneous fission events and then these can go on to cause induced fission events. The distribution reaches a steady state when the neutrons produced in fission are balanced by those being lost through capture events and being leaked from the system. Contrasting this, the distributions in (8b), (8c) and (8d) all start at zero as there are no neutrons in the system initially. The probability for each of these increases as firstly spontaneous and then induced fission events take place. Again, a steady state is reached when the number of neutrons produced in fissions equals those removed from the system by capture and leakage. We note that the probability for finding one neutron in the whole system is much greater than that of finding either two or ten neutrons - the subcriticality of the system and source strength being such that 
retaining a constant population of ten neutrons within the system has a very small probability indeed.

\section{IV.B.2. Surface Counting Region for all Groups and Outward Facing Angles}

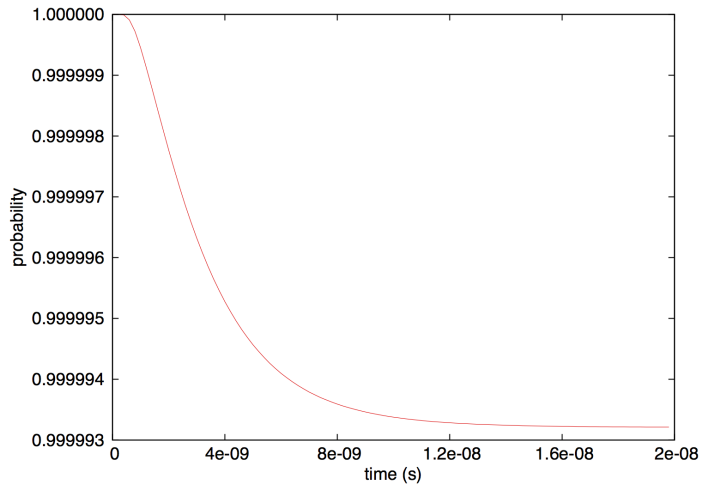

(a) $\theta_{0}$

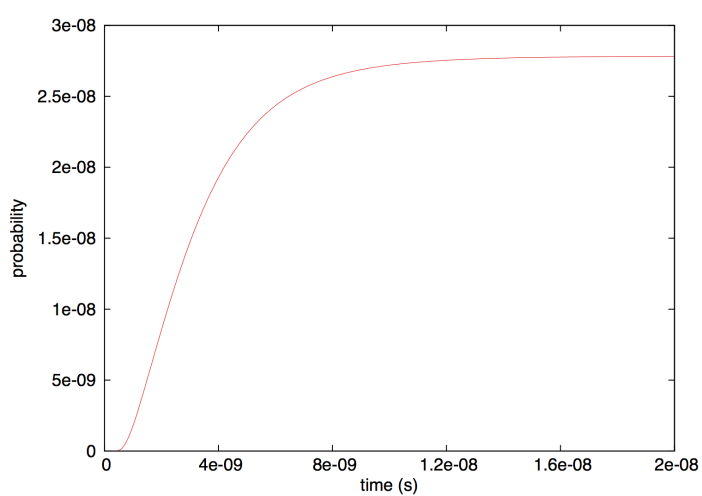

(c) $\theta_{2}$

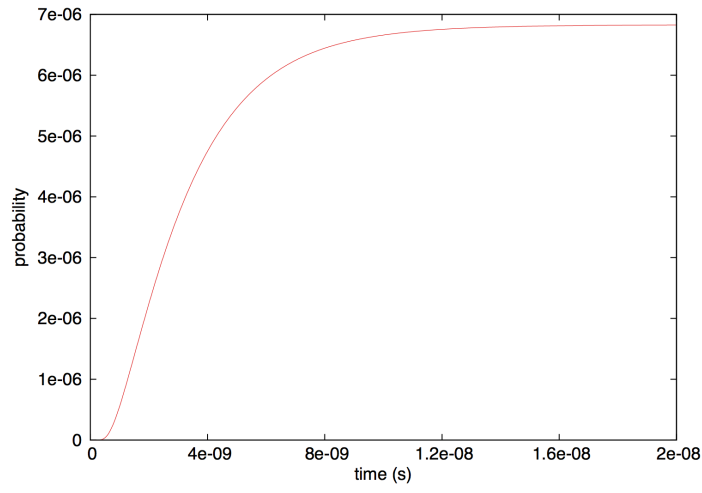

(b) $\theta_{1}$

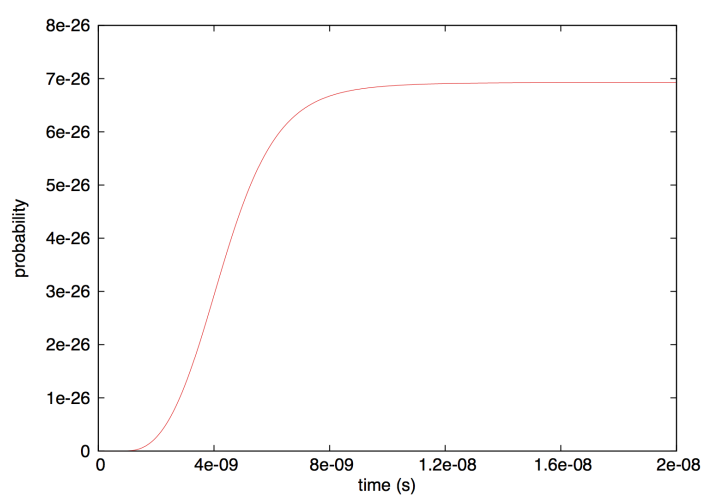

(d) $\theta_{10}$

Figure 9: Probability distribution for finding (9a) zero neutrons, (9b) one neutron, (9c) two neutrons and (9d) ten neutrons in an outward orientation in the boundary cell of the reflector in the presence of a uniform intrinsic source within the multiplying sphere.

The set of figures for a surface counting region shown in figure (9) show the most interesting behaviour in terms of their shape: note that the non-zero probabilities are equal to zero for a small amount of time once the source is switched on. This is because in order to be counted, the neutrons produced in the multiplying sphere have to diffuse across to the counting region. The time taken to do this causes the near-zero region at early times in the distributions. 


\section{Moments}

We now briefly consider the statistical moments of the neutron number probability distribution. These have been dealt with extensively in the literature and are more frequently calculated than the individual number probabilities as they provide a means for establishing key physical properties of the material being investigated $[9,20,11,3]$ and form much simpler equations for solution. Of interest in their own right, the direct computation of these moments also provide an independent assessment of the accuracy of the computed number distributions through comparisons with the moments constructed from the $p_{n}(\vec{r}, E, \vec{\Omega}, s)$ and $\theta_{n}(s)$.

V.A. Statistical Moments for the Probability Distribution of Neutron Number for a Single Initiating Neutron

Differentiating Eq. (2) respect to $z$ setting $z=1$ gives for the factorial moments of the neutron population:

$$
\begin{aligned}
-\left.\frac{\partial^{j} \tilde{G}(z ; \vec{r}, E, \vec{\Omega}, s)}{\partial z^{j}}\right|_{z=1} & =\sum_{n=0}^{\infty} n(n-1) \ldots(n-j+1) p_{n}(\vec{r}, E, \vec{\Omega}, s) \\
& =\overline{n(n-1) \ldots(n-j+1)} \\
& \equiv \bar{N}_{j}(\vec{r}, E, \vec{\Omega}, s)
\end{aligned}
$$

where the dependence of $\tilde{G}$ on $R$ has not been explicitly included. Thus, $\bar{N}_{1}$ is the mean neutron number, $\bar{N}_{2}=\overline{N^{2}}-\bar{N}_{1}$ is related to the variance of the distribution, and so on. In the same way as the equations for the individual number probabilities, the equations for the statistical moments can also be extracted by the differentiation of Eq. (7) with respect to $z$, this time evaluated at $z=1$. This produces a set of coupled equations similar to those for $p_{n}(\vec{r}, E, \vec{\Omega}, s)$ but with one important difference: all the equations in question are linear and inhomogeneous. To illustrate this, consider first the differentiation of Eq. (7) with respect to $z$, giving:

$$
\begin{aligned}
& \frac{1}{v} \frac{\partial}{\partial s}\left(\frac{\partial \tilde{G}(z ; \vec{r}, E, \vec{\Omega}, s)}{\partial z}\right)-\vec{\Omega} \cdot \nabla\left(\frac{\partial \tilde{G}(z ; \vec{r}, E, \vec{\Omega}, s)}{\partial z}\right)+\Sigma_{t}(\vec{r}, E)\left(\frac{\partial \tilde{G}(z ; \vec{r}, E, \vec{\Omega}, s)}{\partial z}\right) \\
& \quad=\int \Sigma_{s}\left(\vec{r}, E \rightarrow E^{\prime}\right)\left[\int\left(\frac{\partial \tilde{G}(z ; \vec{r}, E, \vec{\Omega}, s)}{\partial z}\right) \frac{d \vec{\Omega}^{\prime}}{4 \pi}\right] d E^{\prime} \\
& \quad+\bar{\nu}(\vec{r}, E) \Sigma_{f}(\vec{r}, E)\left[\iint F\left(E^{\prime}\right)\left(\frac{\partial \tilde{G}(z ; \vec{r}, E, \vec{\Omega}, s)}{\partial z}\right) \frac{d \vec{\Omega}^{\prime}}{4 \pi} d E^{\prime}\right] \\
& \quad+\sum_{k=2}^{K} \frac{(-1)^{k}}{(k-1) !} \chi_{k} \Sigma_{f}(\vec{r}, E)\left[\iint F\left(E^{\prime}\right) \tilde{G}(z ; \vec{r}, E, \vec{\Omega}, s) \frac{d \vec{\Omega}^{\prime}}{4 \pi} d E^{\prime}\right]^{k-1} \\
& \quad \times\left[\iint F\left(E^{\prime}\right)\left(\frac{\partial \tilde{G}(z ; \vec{r}, E, \vec{\Omega}, s)}{\partial z}\right) \frac{d \overrightarrow{\Omega^{\prime}}}{4 \pi} d E^{\prime}\right]
\end{aligned}
$$


where we have not included explicitly the dependence of the generating function on the region $R$. If we now evaluate this expression at $z=1$, noting that:

$$
\tilde{G}(z=1 ; \vec{r}, E, \vec{\Omega}, s)=1-\sum_{n=0}^{\infty} p_{n}(\vec{r}, E, \vec{\Omega}, s)=0
$$

we retrieve the standard adjoint transport equation for the mean neutron number:

$$
\begin{aligned}
& \frac{1}{v} \frac{\partial \bar{N}_{1}(\vec{r}, E, \vec{\Omega}, s)}{\partial s}-\vec{\Omega} \cdot \nabla \bar{N}_{1}(\vec{r}, E, \vec{\Omega}, s)+\Sigma_{t}(\vec{r}, E) \bar{N}_{1}(\vec{r}, E, \vec{\Omega}, s)= \\
& \int \Sigma_{s}\left(\vec{r}, E \rightarrow E^{\prime}\right)\left[\int \bar{N}_{1}(\vec{r}, E, \vec{\Omega}, s) \frac{d \vec{\Omega}^{\prime}}{4 \pi}\right] d E^{\prime} \\
& +\bar{\nu}(\vec{r}, E) \Sigma_{f}(\vec{r}, E)\left[\iint F\left(E^{\prime}\right) \bar{N}_{1}(\vec{r}, E, \vec{\Omega}, s) \frac{d \vec{\Omega}^{\prime}}{4 \pi} d E^{\prime}\right]
\end{aligned}
$$

Repeating the process by setting $z=1$ after taking higher derivatives gives, we can write in the general case

$$
\begin{aligned}
& \frac{1}{v} \frac{\partial \bar{N}_{j}(\vec{r}, E, \vec{\Omega}, s)}{\partial s}-\vec{\Omega} \cdot \nabla \bar{N}_{j}(\vec{r}, E, \vec{\Omega}, s)+\Sigma_{t}(\vec{r}, E) \bar{N}_{j}(\vec{r}, E, \vec{\Omega}, s)= \\
& \int \Sigma_{s}\left(\vec{r}, E \rightarrow E^{\prime}\right)\left[\int \bar{N}_{j}(\vec{r}, E, \vec{\Omega}, s) \frac{d \vec{\Omega}^{\prime}}{4 \pi}\right] d E^{\prime} \\
& +\bar{\nu}(\vec{r}, E) \Sigma_{f}(\vec{r}, E)\left[\iint F\left(E^{\prime}\right) \bar{N}_{j}(\vec{r}, E, \vec{\Omega}, s) \frac{d \vec{\Omega}^{\prime}}{4 \pi} d E^{\prime}\right] \\
& +\mathcal{N}\left(\bar{N}_{i}\right)
\end{aligned}
$$

with the initial condition

$$
\bar{N}_{j}(\vec{r}, E, \vec{\Omega}, 0)=\mathcal{I}_{R}(\vec{r}, E, \vec{\Omega}) \delta_{j, 1}
$$

and the boundary condition

$$
\bar{N}_{j}(\vec{r}, E, \vec{\Omega}, s)=0 \quad \vec{r} \in \partial V \quad \vec{e} \cdot \vec{\Omega} \geq 0 \quad j=1,2, \ldots
$$

where $\mathcal{N}\left(\bar{N}_{i}\right)$ is a source term that depends on the lower order moments $\bar{N}_{i}$ where $i<j$. For instance, in the case of the second moment, this source term is given by:

$$
\mathcal{N}\left(\bar{N}_{i}\right)=-\chi_{2} \Sigma_{f}(\vec{r}, E)\left[\iint F\left(E^{\prime}\right) \bar{N}_{1}\left(\vec{r}, E^{\prime}, \vec{\Omega}^{\prime}, s\right) \frac{d \vec{\Omega}^{\prime}}{4 \pi} d E^{\prime}\right]^{2}
$$

which can be continued until sufficiently high moments are calculated. This provides a direct method of calculating the statistical moments of the distributions using the numerical method as detailed for the linear equations in section (IV.A). 
The statistical moments can also be computed from the number probabilities by truncating the sums in Eq. (36) at suitably high $n$ such that the remaining $p_{n}(\vec{r}, E, \vec{\Omega}, s)$ are adequately small. Once the $p_{n}(\vec{r}, E, \vec{\Omega}, s)$ have been calculated, it is then possible to perform the weighted sum in Eq. (36) to establish the approximate statistical moments of the distributions, which if the truncation point has been chosen correctly should show good agreement.

\section{V.A.1. Comparison of Statistical Moments}

We now consider the comparison of the statistical moments of the probability distribution of neutron number in the case of the single initiating neutron. The system investigated is that described in section (IV) of a $3.7938 \mathrm{~cm}$ radius multiplying sphere surrounded by a $1 \mathrm{~cm}$ thick reflector where the counting region extends over all space and angles but only the highest energy group shown in the energy discretisation in tables (II) and (III).

We have performed the truncation of the sums in Eq. (36) at $n=20$, a point at which the number probabilities calculated account for well in excess of $99 \%$ of neutrons within the system. In order to accurately assess the agreement between the two solutions, comparisons for the first six statistical moments have been made, over a range of angles and energy groups. As can be seen in figures (10) and (11) there is a general trend in increasing relative differences for the higher order moments. This is not unexpected as the higher the order of the moment being calculated, the greater is the magnitude of the combinatorial factors in the larger $n$ terms in this sum. Thus, for the higher moments the choice of truncation point becomes more important, requiring the retention of higher order terms more strongly than the lower moments. Despite this, excellent agreement is seen throughout all the moments for the two different means of calculation. 


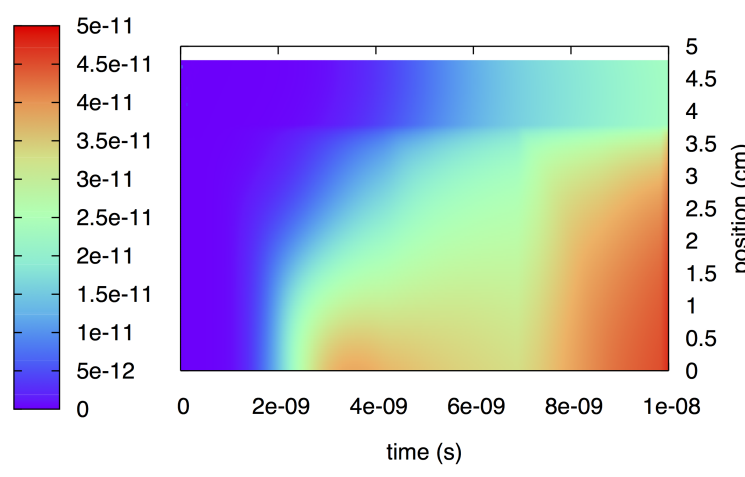

(a) $\bar{N}_{1}$

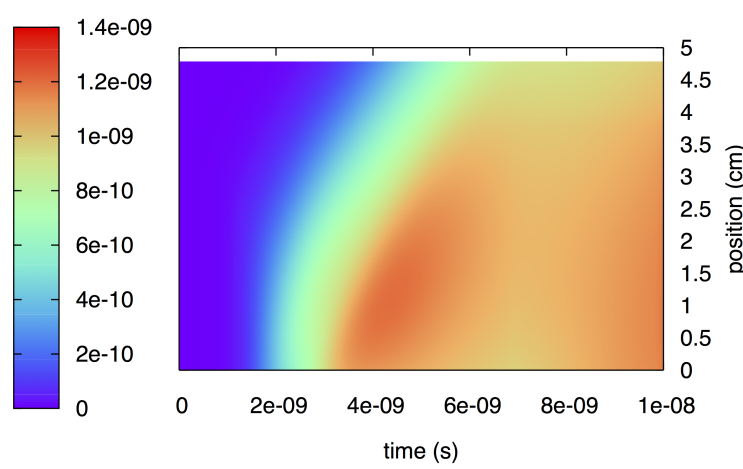

(c) $\bar{N}_{2}$

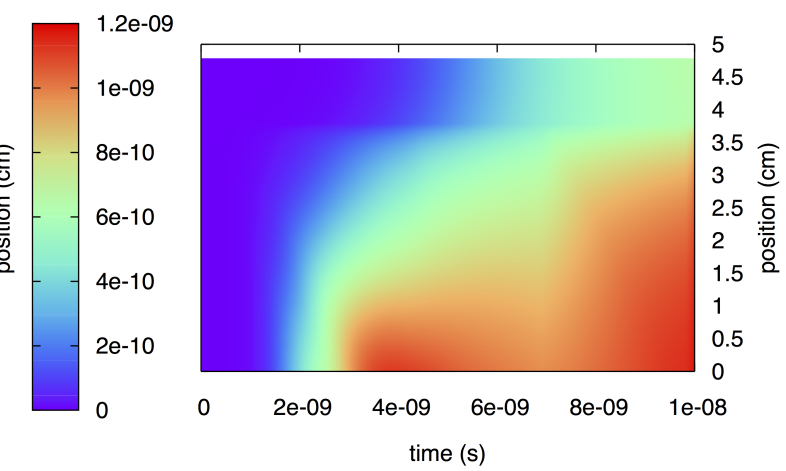

(b) $\bar{N}_{2}$

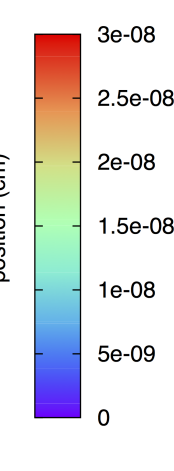

(1)

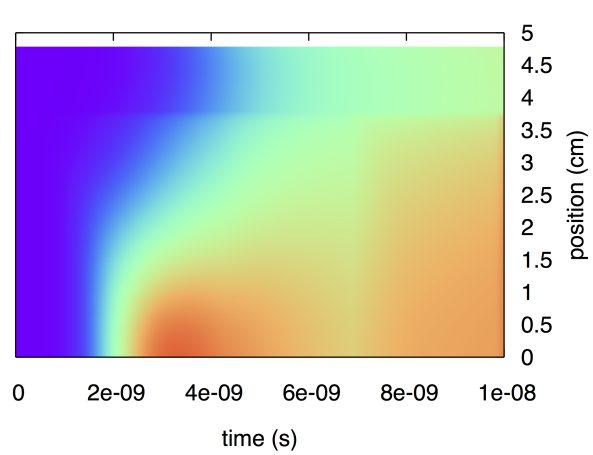

(d) $\bar{N}_{3}$

Figure 10: Relative differences in statistical moments for (10a) the initial neutron injected in group 2 at $\mu=0.5255$, for (10b) the initial neutron injected in group 3 at $\mu=0.7967$, for (10c) the initial neutron injected in group 2 at $\mu=-0.9603$ and for (10d) the initial neutron injected in group 1 at $\mu=0.1834$.

\section{V.B. Statistical Moments for the Probability Distribution of Neutron Number for an Intrinsic Source}

Finally, we consider the statistical moments in the case of an intrinsic random source, which are related to the derivatives of the corresponding generating function by:

$$
\begin{aligned}
-\left.\frac{\partial^{j} G^{(Q)}(s)}{\partial z^{j}}\right|_{z=1} & =\sum_{m=0}^{\infty} m(m-1) \ldots(m-j+1) \theta_{m}(s) \\
& =\overline{m(m-1) \ldots(m-j+1)}(s) \\
& =\bar{M}_{j}(s) .
\end{aligned}
$$

As in the single initiating neutron case, we take the derivatives of Eq. (14) and set $z=1$. Taking the first derivative Eq. (14) gives: 


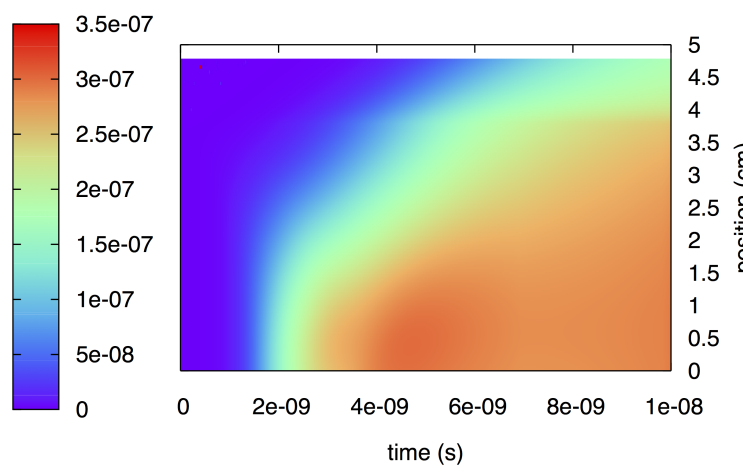

(a) $\bar{N}_{4}$

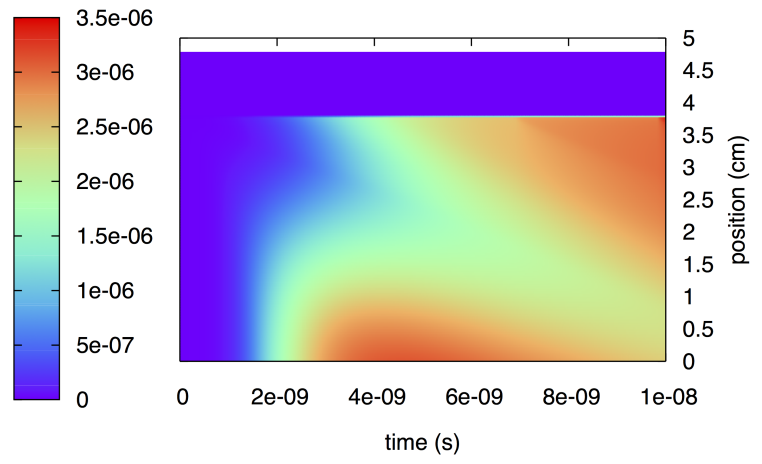

(c) $\bar{N}_{5}$
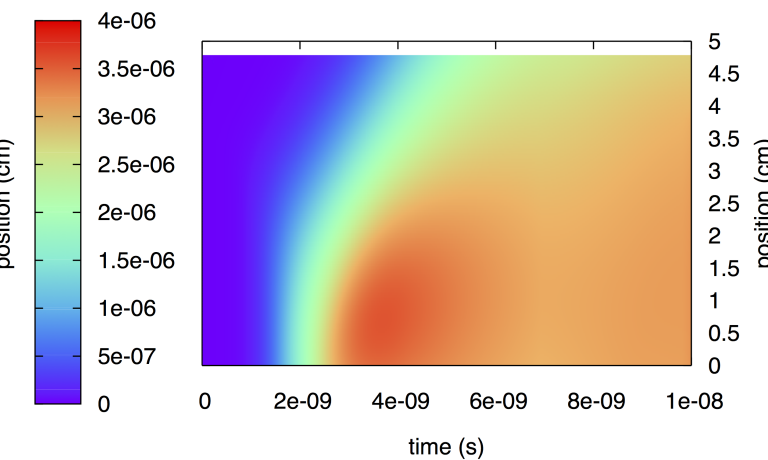

(b) $\bar{N}_{5}$
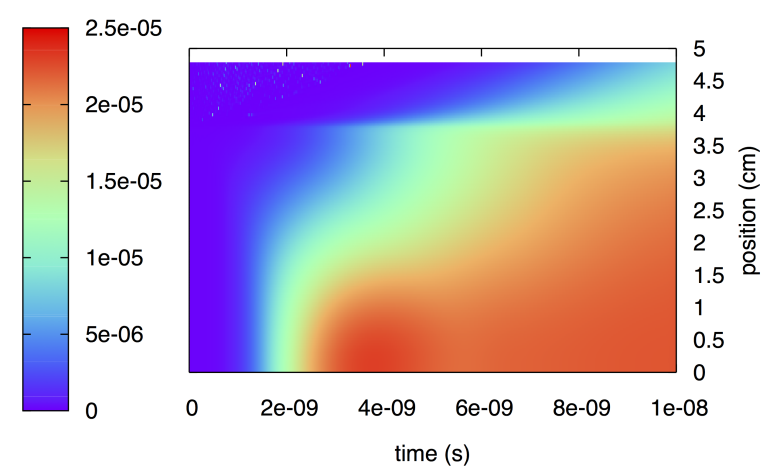

(d) $\bar{N}_{6}$

Figure 11: Relative differences in statistical moments for (11a) the initial neutron injected in group 4 at $\mu=-0.5255$, for (11b) the initial neutron injected in group 2 at $\mu=-0.7967$, for (11c) the initial neutron injected in group 6 at $\mu=0.9603$ and for (11d) the initial neutron injected in group 5 at $\mu=-0.1834$.

$$
\begin{aligned}
& \frac{\partial}{\partial s} \frac{\partial G^{(Q)}(z ; s)}{\partial z}= \\
& Q(\vec{r})\left\{\sum_{k=1}^{K_{Q}} \frac{(-1)^{k}}{k !} \chi_{k}^{(Q)} \int_{V} d V w(\vec{r})\left[\iint F\left(E^{\prime}\right) \tilde{G}\left(z ; \vec{r}, E^{\prime}, \vec{\Omega}^{\prime}, s\right) \frac{d \vec{\Omega}^{\prime}}{4 \pi} d E^{\prime}\right]^{k}\right\} \frac{\partial G^{(Q)}(z ; s)}{\partial z} \\
& +Q(\vec{r}) \sum_{k=1}^{K_{Q}} \frac{(-1)^{k}}{(k-1) !} \chi_{k}^{(Q)} \int_{V} d V w(\vec{r})\left[\iint F\left(E^{\prime}\right) \tilde{G}\left(z ; \vec{r}, E^{\prime}, \vec{\Omega}^{\prime}, s\right) \frac{d \vec{\Omega}^{\prime}}{4 \pi} d E^{\prime}\right]^{k-1} \\
& \times\left[\iint F\left(E^{\prime}\right) \frac{\partial \tilde{G}\left(z ; \vec{r}, E^{\prime}, \vec{\Omega}^{\prime}, s\right)}{\partial z} \frac{d \vec{\Omega}^{\prime}}{4 \pi} d E^{\prime}\right] G^{(Q)}(z ; s)
\end{aligned}
$$

Evaluating this expression at $z=1$, again noting that $\tilde{G}(z=1)=0$, yields an equation relating the mean neutron number in the presence of a random source, $\bar{M}_{1}$, to the mean number for a single chain, $\bar{N}_{1}$ : 


$$
\frac{\partial \bar{M}_{1}(s)}{\partial s}=-Q(\vec{r}) \chi_{1}^{(Q)} \int_{V} d V w(\vec{r})\left[\iint F\left(E^{\prime}\right) \bar{N}_{1}\left(\vec{r}, E^{\prime}, \vec{\Omega}^{\prime}, s\right) \frac{d \vec{\Omega}^{\prime}}{4 \pi} d E^{\prime}\right]
$$

If we repeat this process for the second moment, we obtain

$$
\begin{aligned}
& \frac{\partial \bar{M}_{2}(s)}{\partial s}=-2 Q(\vec{r}) \chi_{1}^{(Q)} \int_{V} d V w(\vec{r})\left[\iint F\left(E^{\prime}\right) \bar{N}_{1}\left(\vec{r}, E^{\prime}, \vec{\Omega}^{\prime}, s\right) \frac{d \vec{\Omega}^{\prime}}{4 \pi} d E^{\prime}\right] \bar{M}_{1}(s) \\
& -Q(\vec{r}) \chi_{1}^{(Q)} \int_{V} d V w(\vec{r})\left[\iint F\left(E^{\prime}\right) \bar{N}_{2}\left(\vec{r}, E^{\prime}, \vec{\Omega}^{\prime}, s\right) \frac{d \vec{\Omega}^{\prime}}{4 \pi} d E^{\prime}\right] \\
& +Q(\vec{r}) \chi_{2}^{(Q)} \int_{V} d V w(\vec{r})\left[\iint F\left(E^{\prime}\right) \bar{N}_{1}\left(\vec{r}, E^{\prime}, \vec{\Omega}^{\prime}, s\right) \frac{d \vec{\Omega}^{\prime}}{4 \pi} d E^{\prime}\right]^{2}
\end{aligned}
$$

and so on. We again compare the moments calculated in this manner to those calculated explicitly using a truncation of Eq. (44) at a suitably high neutron number.

\section{V.B.1. Comparison of Statistical Moments for the Intrinsic Source}

We consider the same assembly as in the single initiating neutron case with the same counting regions. For the moments calculated based on Eq. (44) the series has been truncated at $m=20$. To form an accurate comparison between the two solutions, the first six statistical moments are exhibited here.

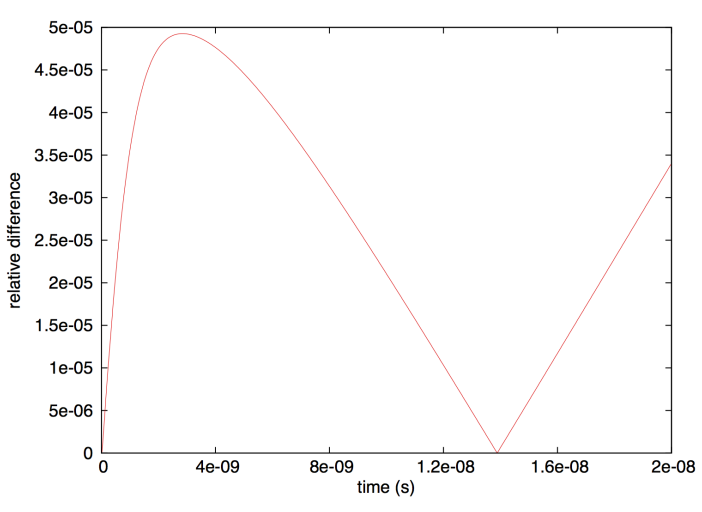

(a) $\bar{M}_{1}$

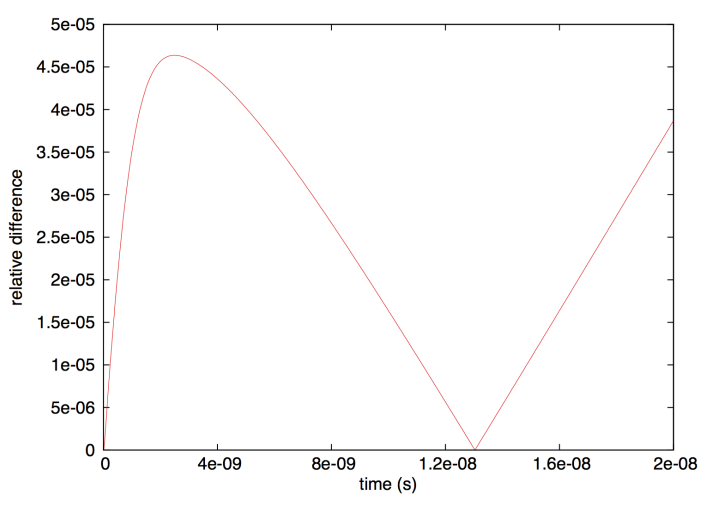

(b) $\bar{M}_{2}$

Figure 12: Relative differences between for the first two statistical moments of the intrinsic source distributions.

As can be observed from the graphs in figures (12) and (13) excellent agreement is seen between the two methods of calculating the statistical moments with the highest relative difference between solutions being in the sixth moment at $8 \times 10^{-5}$. Such small 


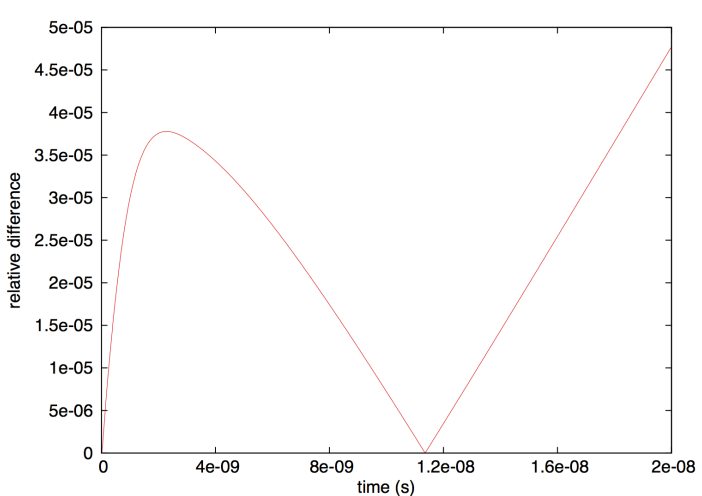

(a) $\bar{M}_{3}$

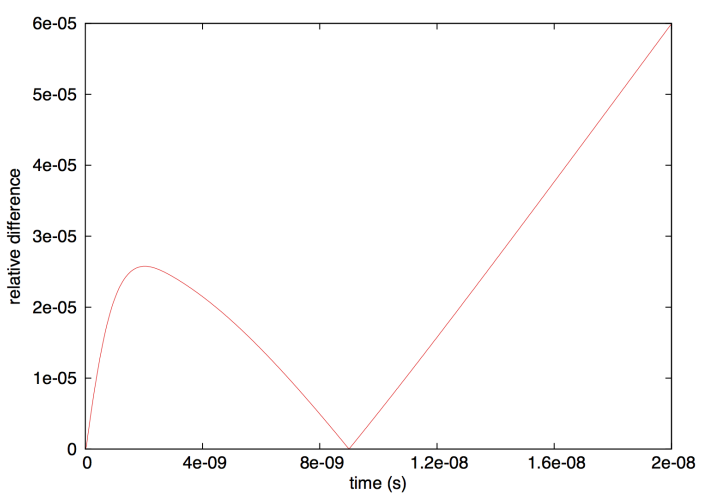

(c) $\bar{M}_{5}$

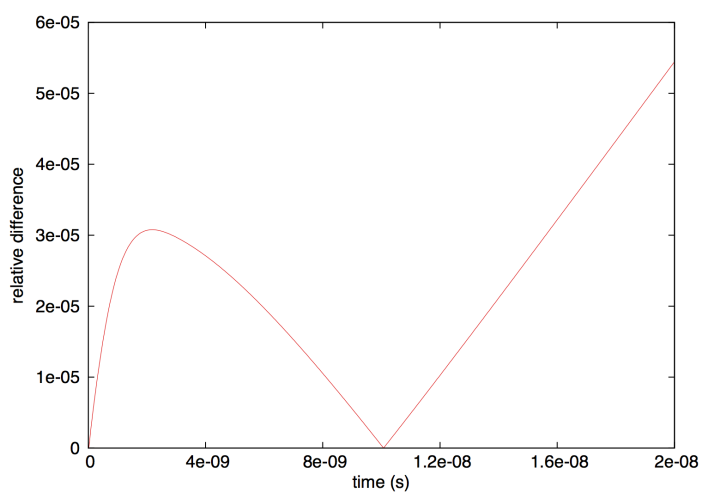

(b) $\bar{M}_{4}$

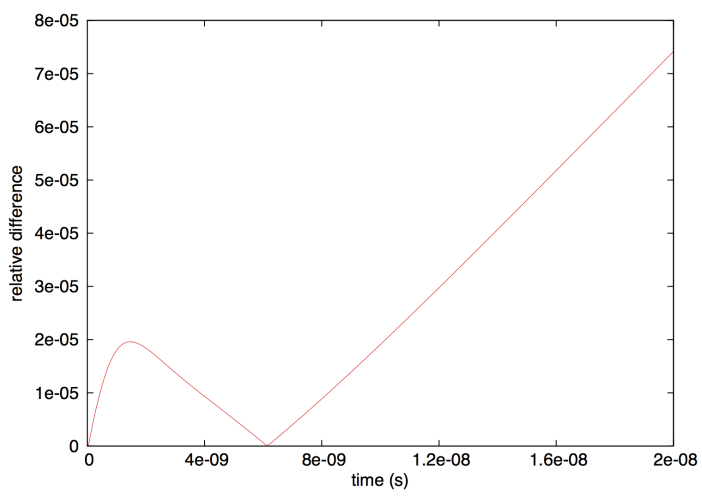

(d) $\bar{M}_{6}$

Figure 13: Relative differences for the third, fourth, fifth and sixth statistical moments of the intrinsic source distributions.

relative differences between the solutions strengthen the validity and accuracy of the numerical results for the number distributions presented above.

\section{Conclusions}

In this paper we have demonstrated that neutron number distributions can be accurately and efficiently computed for subcritical assemblies of interest in nuclear nonproliferation and safeguards research without resorting to a lumped model representation. Our approach, based on the general backward formulation of the generating function, has incorporated temporal, spatial, angular and energy dependence of the neutron population as well as a randomly emitting source. A general scheme for developing the coupled system of equations for the number probabilities was presented and the system solved numerically in one-dimensional spherical geometry using a time-implicit, multigroup, discrete ordinates, and weighted spatial diamond difference scheme, while 
extending our previous work in the one-speed diffusion approximation [4]. Low order statistical moments were also computed, by averaging the number probabilities and applying the same numerical scheme to explicitly derived equations for the moments. The excellent agreement observed between the two different methods of computing the moments, in both the single initiating neutron and intrinsic source cases, provides support for the suitability of the numerical approach adopted for this investigation.

The numerical results have uncovered a range of complex features in the number distributions, which arise from the explicit accounting of neutron energy and direction dependence as well as spatial interfaces and a free surface, and which are suppressed in a lumped model. We see that in the single initiating neutron case there are extremely sharp spatial differences at the boundary between the sphere and the reflector when the initial neutron is injected into the thermal energy group. In general, the inclusion of energy dependence yields solutions with drastically different time progressions and dramatically different spatial features, especially at the interface and surface boundaries. Finally we have seen that the neutron angle dependence also influences the probability distribution, depending on whether the neutron is injected into the fissile region or the reflector, but the angle dependence is most notable when the neutron is introduced near the outer edge of the assembly.

Whilst the work embarked upon here represents a significant extension of work previously undertaken on neutron number probabilities, there is scope for attaining further computational efficiency and adding greater generalisation. The main objective of the present investigation was to demonstrate that neutron number distributions can be computed without sacrificing neutron phase space dependence and without resorting to expensive Monte Carlo simulation, and thereby provide greater information about the multiplying system than is contained in low order statistical averages. As such, it was sufficient for the purposes of this study to employ standard numerical schemes that were adequate for the spherically symmetric geometry considered here without any regard to optimality of these methods. The use of advanced numerical algorithms and programming techniques, or indeed adapting existing production transport codes that are optimised for accuracy and performance, will clearly make feasible the application of this approach to more complex geometries that require multiple spatial dimensions to describe, and enable computation of number probabilities of even higher order. Gains in computational efficiency will also offset the cost of greater mathematical complexity associated with generalising the Master equation to also accommodate photon distributions within the assembly as well as distributions in detectors external to the assembly. We hope to report on such generalisations in future publications.

\section{Acknowledgements}

Joanna Saxby would like to acknowledge the support of EPSRC under their industrial doctorate programme (EPSRC grant number: EP/G037426/1) as well as industrial support from the Atomic Weapons Establishment (AWE), Aldermaston, United Kingdom. 
Anil K. Prinja would like to acknowledge the support of the Nuclear Engineering Group at Imperial College, London.

M.D. Eaton would like to would like to thank EPSRC for their support through the following grants: "Adaptive Hierarchical Radiation Transport Methods to Meet Future Challenges in Reactor Physics (EPSRC grant number: EP/J002011/1) and "RADIANT: A Parallel, Scalable, High Performance Radiation Transport Modelling and Simulation Framework for Reactor Physics, Nuclear Criticality Safety Assessment and Radiation Shielding Analyses (EPSRC grant number: EP/K503733/1).

\section{References}

[1] E C Miller, J K Mattingly, S D Clarke, C J Solomon, B Dennis, A Meldrum, and S A Pozzi. Computational evaluation of neutron multiplicity measurements of polyethylene-reflected plutonium metal. Nuclear Science and Engineering, 176:167-185, 2014.

[2] A Enqvist, I Pázsit, and S Pozzi. The number distribution of neutrons and gamma photon generated in a multiplying sample. Nuclear Instruments $\&$ Methods in Physics Research, Section A: Accelerators, Spectrometers, Detectors, and Associated Equipment, 566:598-608, 2006.

[3] John Mattingly. Computation of neutron multiplicity statistics using deterministic transport. IEEE Transactions on Nuclear Science, 59:314-322, 2012.

[4] J E M Saxby, A K Prinja, and M D Eaton. Diffusion theory model of the neutron number probability distribution in a subcritical multiplying assembly. Annals of Nuclear Energy, 109:507-528, 2017.

[5] J E M Saxby, M M R Williams, and M D Eaton. The energy dependent Pál-Bell equation and the influence of the neutron energy on the survival probability in a supercritical medium. Annals of Nuclear Energy, 92:413-418, 2016.

[6] Joseph Abate and Ward Whitt. Numerical inversion of probability generating functions. Operational Research Letters, 12:245-251, 1992.

[7] M M R Williams. Burst wait-times in the caliban reactor using the gamma probability distribution function. Nuclear Science and Engineering, 183:116-125, 2016.

[8] David H Chambers, Hema Chandrasekaran, and Sean E Walston. Fourier method for calculating fission chain neutron multiplicity distributions. Nuclear Science and Engineering, 184:244-253, 2016.

[9] George I Bell. On the stochastic theory of neutron transport. Nuclear Science and Engineering, 21:390 - 401, 1965.

[10] L Pál. Statistical theory of the chain reaction in nuclear Reactors: Parts I, II, III. Technical report, Atomic Energy Authority, 1962. 
[11] I Pázsit and L Pál. Neutron Fluctuations: A Treatise on the Physics of Branching Processes. Elsevier, 2008.

[12] J Lewins. Linear stochastic neutron transport theory. Proceedings of the Royal Society of London A, 362:537-558, 1978.

[13] K Böhnel. The effect of multiplication on the quantitative determination of spontaneously fissioning isotopes by neutron correlation analysis. Nuclear Science and Engineering, 90:75, 1985.

[14] Alain Hebert. Applied Reactor Physics. Presses Internationales Polytechnique, 2009.

[15] William F Ames. Numerical Methods for Partial Differential Equations. Academic Press, 1977.

[16] J E Morel and G R Montry. Analysis and elimination of the discrete-ordinates flux dip. Transport Theory and Statistical Physics, 13:5:615-633, 1984.

[17] R Baker. Deterministic methods for time-dependent stochastic neutron transport. Technical Report LA-UR-09-0/057, Los Alamos National Laboratory, 2009.

[18] WIMS. WIMS: A Modular Scheme for Neutronics Calculations. AMEC FOSTER WHEELER, 2014. User Guide for Version 10 Issue 1.

[19] G E Hansen and W H Roach. Six and sixteen group cross sections for fast and intermediate critical assemblies. Technical report, Los Alamos National Laboratory, 1961.

[20] J L Munoz-Cobo, R B Perez, and G Verdu. Stochastic neutron transport theory: Neutron counting statistics in nuclear assemblies. Nuclear Science and Engineering, 95:83 - 105, 1987. 


\section{Appendix A. An Algorithm to Generate the Neutron Number Probability Equations}

Having looked at the individual equations for the number probabilities, both in the case of a single initiating neutron and an intrinsic source, it clear that the complexity associated with using the backward approach is encapsulated in the terms produced from the derivatives of the non-linear term in Eq. (7). As a result, in this appendix, we give details of the algorithm developed in order to produce the equations for solution when considering the neutron number probabilities of general order.

\section{Appendix A.A. Single Initiating Neutron}

Consider Eqs. (19) and (20). For the most part, these equations have very similar terms: we have a general adjoint transport equation in either $p_{1}(\vec{r}, E, \vec{\Omega}, s)$ or $p_{2}(\vec{r}, E, \vec{\Omega}, s)$ with additional terms created from the differentiation of the non-linear term in Eq. (7). It is these additional terms, which effectively act as a source within the general transport equation, which need to be modelled by the algorithm created.

Consider, now, the differentiation of the non-linear term in Eq. (7) as given by

$$
\begin{aligned}
\frac{\partial}{\partial z} & {\left[\Sigma_{f}(\vec{r}, E) \sum_{k=2}^{K} \frac{(-1)^{k}}{k !} \chi_{k}\left[\iint F\left(E^{\prime}\right) \tilde{G}\left(R, z ; \vec{r}, E^{\prime}, \vec{\Omega}^{\prime}, s\right) d E^{\prime} \frac{d \overrightarrow{\Omega^{\prime}}}{4 \pi}\right]^{k}\right] } \\
& =\frac{\partial}{\partial z}\left[\Sigma_{f}(\vec{r}, E) \sum_{k=2}^{K} \frac{(-1)^{k}}{k !} \chi_{k} P(\tilde{G}(z))^{k}\right] \\
& =\frac{\partial}{\partial z}[K(P(\tilde{G}(z)))]
\end{aligned}
$$

To differentiate Eq. (A.1), requires the use of a chain rule, via

$$
\begin{aligned}
\frac{\partial}{\partial z}[K(P(\tilde{G}(z)))] & =\frac{\partial K}{\partial P} \frac{\partial P}{\partial \tilde{G}} \frac{\partial \tilde{G}}{\partial z} \\
& =\Sigma_{f}(\vec{r}, E) \sum_{k=2}^{K} \frac{(-1)^{k}}{k !} \chi_{k} \\
& \times k\left[\iint F\left(E^{\prime}\right) \tilde{G}\left(R, z ; \vec{r}, E^{\prime}, \vec{\Omega}^{\prime}, s\right) d E^{\prime} \frac{d \overrightarrow{\Omega^{\prime}}}{4 \pi}\right]^{k-1} \\
& \times\left[\iint F\left(E^{\prime}\right) \frac{\partial \tilde{G}\left(R, z ; \vec{r}, E^{\prime}, \overrightarrow{\Omega^{\prime}}, s\right)}{\partial z} d E^{\prime} \frac{d \overrightarrow{\Omega^{\prime}}}{4 \pi}\right]
\end{aligned}
$$

For the purpose of the algorithm, we re-write this as 


$$
\begin{aligned}
\frac{\partial}{\partial z}[K(P(\tilde{G}(z)))] & =\frac{\partial K}{\partial P} \frac{\partial P}{\partial \tilde{G}} \frac{\partial \tilde{G}}{\partial z} \\
& =\Sigma_{f}(\vec{r}, E) \sum_{k=2}^{K} \frac{(-1)^{k}}{(k-1) !} \chi_{k}\left[\iint F\left(E^{\prime}\right) \tilde{G}\left(R, z ; \vec{r}, E^{\prime}, \vec{\Omega}^{\prime}, s\right) d E^{\prime} \frac{d \overrightarrow{\Omega^{\prime}}}{4 \pi}\right]^{k-1} \\
& \times\left[\iint F\left(E^{\prime}\right) \frac{\partial \tilde{G}\left(R, z ; \vec{r}, E^{\prime}, \overrightarrow{\Omega^{\prime}}, s\right)}{\partial z} d E^{\prime} \frac{d \overrightarrow{\Omega^{\prime}}}{4 \pi}\right]
\end{aligned}
$$

and note that the first line of this, strongly resembles the original function, $K$ whilst the second line strongly resembles $P$. We, now re-write the terms as

$$
\begin{aligned}
K(P(\tilde{G})) & =\mathcal{K}(0)=\sum_{k=2}^{K} \frac{(-1)^{k}}{k !} \chi_{k}\left[\iint F\left(E^{\prime}\right) \tilde{G}\left(R, z ; \vec{r}, E^{\prime}, \overrightarrow{\Omega^{\prime}}, s\right) d E^{\prime} \frac{d \overrightarrow{\Omega^{\prime}}}{4 \pi}\right]^{k} \\
P(\tilde{G}) & =\mathcal{P}(0)=\left[\iint F\left(E^{\prime}\right) \tilde{G}\left(R, z ; \vec{r}, E^{\prime}, \overrightarrow{\Omega^{\prime}}, s\right) d E^{\prime} \frac{d \overrightarrow{\Omega^{\prime}}}{4 \pi}\right]
\end{aligned}
$$

Noting the resemblance to the original functions, we then write the derivative terms as

$$
\begin{aligned}
& \mathcal{K}(1)=\sum_{k=2}^{K} \frac{(-1)^{k}}{(k-1) !} \chi_{k}\left[\iint F\left(E^{\prime}\right) \tilde{G}\left(R, z ; \vec{r}, E^{\prime}, \overrightarrow{\Omega^{\prime}}, s\right) d E^{\prime} \frac{d \overrightarrow{\Omega^{\prime}}}{4 \pi}\right]^{k-1} \\
& \mathcal{P}(1)=\left[\iint F\left(E^{\prime}\right) \frac{\partial \tilde{G}\left(R, z ; \vec{r}, E^{\prime}, \overrightarrow{\Omega^{\prime}}, s\right)}{\partial z} d E^{\prime} \frac{d \overrightarrow{\Omega^{\prime}}}{4 \pi}\right]
\end{aligned}
$$

and so on, such that generally

$$
\begin{aligned}
& \mathcal{K}(j)=\sum_{k=2}^{K} \frac{(-1)^{k}}{(k-j) !} \chi_{k}\left[\iint F\left(E^{\prime}\right) \tilde{G}\left(R, z ; \vec{r}, E^{\prime}, \overrightarrow{\Omega^{\prime}}, s\right) d E^{\prime} \frac{d \overrightarrow{\Omega^{\prime}}}{4 \pi}\right]^{k-j} \\
& \mathcal{P}(j)=\left[\iint F\left(E^{\prime}\right) \frac{\partial^{j} \tilde{G}\left(R, z ; \vec{r}, E^{\prime}, \overrightarrow{\Omega^{\prime}}, s\right)}{\partial z^{j}} d E^{\prime} \frac{d \overrightarrow{\Omega^{\prime}}}{4 \pi}\right]
\end{aligned}
$$

so that Eq. (A.3) can be compactly re-written as

$$
\frac{\partial}{\partial z} \mathcal{K}(0)=\mathcal{K}(1) \mathcal{P}(1)
$$

In this way, when differentiated with respect to $z$, these two types of terms have the following relationships, for $\mathcal{K}(j)$ 


$$
\begin{aligned}
\frac{\partial}{\partial z} \mathcal{K}(1) & =\mathcal{K}(2) \mathcal{P}(1) \\
\frac{\partial}{\partial z} \mathcal{K}(2) & =\mathcal{K}(3) \mathcal{P}(1) \\
& \cdots \\
\frac{\partial}{\partial z} \mathcal{K}(j) & =\mathcal{K}(j+1) \mathcal{P}(1)
\end{aligned}
$$

and for $\mathcal{P}(j)$

$$
\begin{aligned}
\frac{\partial}{\partial z} \mathcal{P}(1) & =\mathcal{P}(2) \\
\frac{\partial}{\partial z} \mathcal{P}(2) & =\mathcal{P}(3) \\
& \cdots \\
\frac{\partial}{\partial z} \mathcal{P}(j) & =\mathcal{P}(j+1)
\end{aligned}
$$

If products of these two types of term are being differentiated, the product rule can be trivially applied as is the case when considering the $n^{\text {th }}$ derivatives of the function $\mathcal{K}(0)$. The first part of the algorithm performs exactly this for the $(n+1)^{t h}$ derivative by "reading" a term from the $n^{\text {th }}$ derivative and then systematically working through each constituent of the product and applying the rules detailed in Eqs. (A.8) and (A.9) whilst keeping the other constituents of the product the same. Performing this, the first step of the algorithm generates

$$
\begin{aligned}
\frac{\partial}{\partial z} \mathcal{K}(0) & =\mathcal{K}(1) \mathcal{P}(1) \\
\frac{\partial^{2}}{\partial z^{2}} \mathcal{K}(0) & =\mathcal{K}(2) \mathcal{P}(1) \mathcal{P}(1)+\mathcal{K}(1) \mathcal{P}(2) \\
\frac{\partial^{3}}{\partial z^{3}} \mathcal{K}(0) & =\mathcal{K}(3) \mathcal{P}(1) \mathcal{P}(1) \mathcal{P}(1)+\mathcal{K}(2) \mathcal{P}(2) \mathcal{P}(1)+\mathcal{K}(2) \mathcal{P}(1) \mathcal{P}(2) \\
& +\mathcal{K}(2) \mathcal{P}(1) \mathcal{P}(2)+\mathcal{K}(1) \mathcal{P}(3)
\end{aligned}
$$

where the scale of escalation of the number of terms, becomes clear for each successive differentiation. In light of this, it is obvious that like terms should be collected, thus the next step of the algorithm orders the terms as

$$
\begin{aligned}
\frac{\partial}{\partial z} \mathcal{K}(0) & =\mathcal{K}(1) \mathcal{P}(1) \\
\frac{\partial^{2}}{\partial z^{2}} \mathcal{K}(0) & =\mathcal{K}(2) \mathcal{P}(1) \mathcal{P}(1)+\mathcal{K}(1) \mathcal{P}(2) \\
\frac{\partial^{3}}{\partial z^{3}} \mathcal{K}(0) & =\mathcal{K}(3) \mathcal{P}(1) \mathcal{P}(1) \mathcal{P}(1)+\mathcal{K}(2) \mathcal{P}(2) \mathcal{P}(1)+\mathcal{K}(2) \mathcal{P}(2) \mathcal{P}(1) \\
& +\mathcal{K}(2) \mathcal{P}(2) \mathcal{P}(1)+\mathcal{K}(1) \mathcal{P}(3)
\end{aligned}
$$


so that the third step of the algorithm can collect like terms as

$$
\begin{aligned}
\frac{\partial}{\partial z} \mathcal{K}(0) & =\mathcal{K}(1) \mathcal{P}(1) \\
\frac{\partial^{2}}{\partial z^{2}} \mathcal{K}(0) & =\mathcal{K}(2) \mathcal{P}(1) \mathcal{P}(1)+\mathcal{K}(1) \mathcal{P}(2) \\
\frac{\partial^{3}}{\partial z^{3}} \mathcal{K}(0) & =\mathcal{K}(3) \mathcal{P}(1) \mathcal{P}(1) \mathcal{P}(1)+3 \mathcal{K}(2) \mathcal{P}(2) \mathcal{P}(1)+\mathcal{K}(1) \mathcal{P}(3)
\end{aligned}
$$

The $\mathcal{K}, \mathcal{P}$ and coefficient terms are then stored in three two-dimensional matrices such that the $n^{\text {th }}$ row represents the terms of the $n^{\text {th }}$ derivative with new terms in the summation being indicated by the presence of a 0 . As such, if we were to re-write the terms in Eq. (A.12) in these three matrices $\mathbb{K}, \mathbb{P}$ and $\mathbb{C}$ they would take the following form

$$
\begin{gathered}
\mathbb{P}=\left(\begin{array}{llllllll}
1 & 0 & 0 & 0 & 0 & 0 & 0 & 0 \\
1 & 1 & 0 & 2 & 0 & 0 & 0 & 0 \\
1 & 1 & 1 & 0 & 2 & 1 & 0 & 3
\end{array}\right) \quad \mathbb{K}=\left(\begin{array}{lllllllll}
1 & 0 & 0 & 0 & 0 & 0 & 0 & 0 \\
2 & 0 & 0 & 1 & 0 & 0 & 0 & 0 \\
3 & 0 & 0 & 0 & 2 & 0 & 0 & 1
\end{array}\right) \\
\mathbb{C}=\left(\begin{array}{llllllll}
1 & 0 & 0 & 0 & 0 & 0 & 0 & 0 \\
1 & 0 & 0 & 1 & 0 & 0 & 0 & 0 \\
1 & 0 & 0 & 0 & 3 & 0 & 0 & 1
\end{array}\right)
\end{gathered}
$$

As a verification of the validity of the algorithm constructed, the first eight equations were compared to those in table A.I of [13] and were in agreement. Note that in order to transform these equations for the differentials of the generating function into equations for the neutron number probabilities, these expressions need to be evaluated at $z=0$ which has the result of changing the signs of the terms in the summation, dependent on the $\mathcal{K}$ term: odd numbers here equate to a minus and even numbers to a plus.

In the case of the intrinsic source, a similar process was undertaken. Consider now Eq. (14). Similarly to the single initiating neutron case, this has a nested part - with the same structure as $\mathcal{K}$ which will create new $\mathcal{K}$ terms - which we note are not identical to the $\mathcal{K}$ terms in the single initiating neutron case - and an additional $\mathcal{P}$ term, but this time it is multiplied by a $\Theta$ term. The form of the equation to be differentiated is therefore similar to

$$
\frac{\partial}{\partial z} \mathcal{K}(0) \Theta(0)=\frac{\partial \mathcal{K}(0)}{\partial z} \Theta(0)+\mathcal{K}(0) \frac{\partial \Theta(0)}{\partial z}
$$

where this time the $\mathcal{K}$ terms are defined as

$$
\mathcal{K}(j)=\sum_{k=1}^{K_{Q}} \frac{(-1)^{k}}{(k-j) !} \chi_{k}^{(Q)} \int w(\vec{r})\left[\iint F\left(E^{\prime}\right) \tilde{G}\left(R, z ; \vec{r}, E^{\prime}, \vec{\Omega}, s\right) d E^{\prime} \frac{d \vec{\Omega}^{\prime}}{4 \pi}\right]^{(k-j)}
$$


and undertake the same pattern in differentiation as in Eq. (A.11), and where we have used a simple product rule. Additionally we note that

$$
\begin{gathered}
\frac{\partial}{\partial z} \Theta(0)=\Theta(1)=\frac{\partial G^{(Q)}}{\partial z} \\
\frac{\partial}{\partial z} \Theta(1)=\Theta(2)=\frac{\partial^{2} G^{(Q)}}{\partial z^{2}} \\
\ldots \\
\frac{\partial}{\partial z} \Theta(j)=\Theta(j+1)=\frac{\partial^{(j+1)} G^{(Q)}}{\partial z^{(j+1)}}
\end{gathered}
$$

so that Eq. (A.14) can be written as

$$
\frac{\partial}{\partial z} \mathcal{K}(0) \Theta(0)=\mathcal{K}(1) \mathcal{P}(1) \Theta(0)+\mathcal{K}(0) \Theta(1)
$$

similarly the next few derivatives become

$$
\begin{aligned}
\frac{\partial^{2}}{\partial z^{2}} \mathcal{K}(0) \Theta(0) & =\mathcal{K}(2) \mathcal{P}(1) \mathcal{P}(1) \Theta(0)+\mathcal{K}(1) \mathcal{P}(2) \Theta(0)+2 \mathcal{K}(1) \mathcal{P}(1) \Theta(1) \\
& +\mathcal{K}(0) \Theta(2) \\
\frac{\partial^{3}}{\partial z^{3}} \mathcal{K}(0) \Theta(0) & =\mathcal{K}(3) \mathcal{P}(1) \mathcal{P}(1) \mathcal{P}(1) \Theta(0)+3 \mathcal{K}(2) \mathcal{P}(2) \mathcal{P}(1) \Theta(0) \\
& +3 \mathcal{K}(2) \mathcal{P}(1) \mathcal{P}(1) \Theta(1)+\mathcal{K}(1) \mathcal{P}(3) \Theta(0)+3 \mathcal{K}(1) \mathcal{P}(2) \Theta(1) \\
& +2 \mathcal{K}(1) \mathcal{P}(1) \Theta(2)+\mathcal{K}(0) \Theta(3)
\end{aligned}
$$

By the comparison of Eq. (A.18) with (A.12), it is clear that the equations are much more lengthy in the case of the intrinsic source. The algorithm created no longer works through differentiation and collection but is simplified by noting that the terms which contain $\Theta(0)$ are the same as the terms in Eq. (A.12), but with the addition of a $\Theta(0)$ term, and that for the remaining terms, only the $\Theta(j)$ terms from the previous order have to be increased to $\Theta(j+1)$. Consequently the algorithm copies terms from the corresponding order in the single initiating neutron case, adding a $\Theta(0)$ and then copies terms from the previous intrinsic source order, increasing the $\Theta(j)$ by one. This process requires an additional matrix to store the $\Theta$ terms and as $\Theta(0)$ terms are now present, new terms are indicated in the intrinsic source by a high number, much greater than the order being calculated. The resultant equations were checked against the first ten hand derived equations and were consistent. 